\title{
HOW TO GET A CONSERVATIVE WELL-POSED LINEAR SYSTEM OUT OF THIN AIR. PART I. WELL-POSEDNESS AND ENERGY BALANCE
}

\author{
George Weiss ${ }^{1}$ And Marius TucsnaK ${ }^{2}$
}

\begin{abstract}
Let $A_{0}$ be a possibly unbounded positive operator on the Hilbert space $H$, which is boundedly invertible. Let $C_{0}$ be a bounded operator from $\mathcal{D}\left(A_{0}^{\frac{1}{2}}\right)$ to another Hilbert space $U$. We
\end{abstract} prove that the system of equations

$$
\begin{gathered}
\ddot{z}(t)+A_{0} z(t)+\frac{1}{2} C_{0}^{*} C_{0} \dot{z}(t)=C_{0}^{*} u(t), \\
y(t)=-C_{0} \dot{z}(t)+u(t),
\end{gathered}
$$

determines a well-posed linear system with input $u$ and output $y$. The state of this system is

$$
x(t)=\left[\begin{array}{c}
z(t) \\
\dot{z}(t)
\end{array}\right] \in \mathcal{D}\left(A_{0}^{\frac{1}{2}}\right) \times H=X
$$

where $X$ is the state space. Moreover, we have the energy identity

$$
\|x(t)\|_{X}^{2}-\|x(0)\|_{X}^{2}=\int_{0}^{T}\|u(t)\|_{U}^{2} \mathrm{~d} t-\int_{0}^{T}\|y(t)\|_{U}^{2} \mathrm{~d} t
$$

We show that the system described above is isomorphic to its dual, so that a similar energy identity holds also for the dual system and hence, the system is conservative. We derive various other properties of such systems and we give a relevant example: a wave equation on a bounded $n$-dimensional domain with boundary control and boundary observation on part of the boundary.

Mathematics Subject Classification. 93C25, 93C20, 35B37.

Received January 17, 2003.

\section{INTRODUCTION AND MAIN RESULTS}

The aim of this work is to show how to construct a conservative linear system from two very simple ingredients: a self-adjoint positive and boundedly invertible operator $A_{0}$ on a Hilbert space $H$ and a bounded operator $C_{0}$

Keywords and phrases. Well-posed linear system, operator semigroup, dual system, energy balance equation, conservative system, wave equation.

1 Department of Electrical and Electronic Engineering, Imperial College London, Exhibition Road, London SW7 2BT, UK; e-mail: G.Weiss@imperial.ac.uk

2 Department of Mathematics, University of Nancy I, BP. 239, 54506 Vandœuvre-les-Nancy, France;

e-mail: Marius.Tucsnak@iecn.u-nancy.fr 
from the domain of $A_{0}^{\frac{1}{2}}$ into another Hilbert space $U$. It turns out that our construction appears naturally in mathematical models of vibrating systems with damping.

By a well-posed linear system we mean a linear time-invariant system $\Sigma$ such that on any finite time interval $[0, \tau]$, the operator $\Sigma_{\tau}$ from the initial state $x(0)$ and the input function $u$ to the final state $x(\tau)$ and the output function $y$ is bounded. The input, state and output spaces are Hilbert spaces, and the input and output functions are of class $L_{\mathrm{loc}}^{2}$. For any $u \in L_{\text {loc }}^{2}$ and any $\tau \geq 0$, we denote by $\mathbf{P}_{\tau} u$ its truncation to the interval $[0, \tau]$. Then the well-posed system $\Sigma$ consists of the family of bounded operators $\Sigma=\left(\Sigma_{\tau}\right)_{\tau \geq 0}$ such that

$$
\left[\begin{array}{l}
x(\tau) \\
\mathbf{P}_{\tau} y
\end{array}\right]=\Sigma_{\tau}\left[\begin{array}{l}
x(0) \\
\mathbf{P}_{\tau} u
\end{array}\right]
$$

For the detailed definition, background and examples we refer to Salamon [15,16], Staffans [17,18], Weiss [24,25] and Weiss and Rebarber [26]. We follow the notation and terminology of [24-26]. Some background on wellposed systems and related concepts will be given in Section 3. The well-posed linear system $\Sigma$ is called conservative if for every $\tau \geq 0, \Sigma_{\tau}$ is unitary. Denoting the state space of $\Sigma$ by $X$, its input space by $U$ and its output space by $Y$, the fact that $\Sigma$ is conservative means that for every $\tau \geq 0$, the following two statements hold:

(i) $\Sigma_{\tau}$ is an isometry, i.e.,

$$
\|x(\tau)\|^{2}+\int_{0}^{\tau}\|y(t)\|^{2} \mathrm{~d} t=\|x(0)\|^{2}+\int_{0}^{\tau}\|u(t)\|^{2} \mathrm{~d} t
$$

(ii) $\Sigma_{\tau}$ is onto, which means that for every $x(\tau) \in X$ and every $\mathbf{P}_{\tau} y \in L^{2}([0, \tau], Y)$, we can find $x(0) \in X$ and $\mathbf{P}_{\tau} u \in L^{2}([0, \tau], U)$ such that (1.1) holds.

Our concept of a conservative linear system is equivalent to what Arov and Nudelman [1] call a conservative scattering system and it goes back to the work of Lax and Phillips [10]. A recent survey paper covering also conservative systems (with some new material) is Weiss et al. [27].

To get a better feeling for the concept of a conservative linear system, consider the simple case when $\Sigma$ is finite-dimensional, i.e., described by

$$
\left\{\begin{array}{l}
\dot{x}(t)=A x(t)+B u(t) \\
y(t)=C x(t)+D u(t)
\end{array}\right.
$$

with $A, B, C$ and $D$ matrices of appropriate dimensions and $t \geq 0$. Then $\Sigma$ is conservative if and only if these matrices satisfy

$$
A+A^{*}=-C^{*} C, \quad B=-C^{*} D, \quad D^{*} D=I, \quad D D^{*}=I
$$

(these imply $A+A^{*}=-B B^{*}$ and $C=-D B^{*}$ ), see [1] (p. 16). If the finite-dimensional $\Sigma$ is conservative, then its transfer function $\mathbf{G}(s)=C(s I-A)^{-1} B+D$ is bounded and analytic on the open right half-plane $\mathbb{C}_{0}$ and for all $\omega \in \mathbb{R}$,

$$
\mathbf{G}^{*}(i \omega) \mathbf{G}(i \omega)=\mathbf{G}(i \omega) \mathbf{G}(i \omega)^{*}=I .
$$

(In a more technical language, $\mathbf{G}$ is inner and co-inner.)

Now let us move to a slightly higher level of generality, namely to well-posed linear systems with bounded $B$ and $C$. This means that $U, X$ and $Y$ are Hilbert spaces and the system is described by (1.3), where $A$ is the generator of strongly continuous semigroup of operators $\mathbb{T}$ on the Hilbert space $X, B \in \mathcal{L}(U, X), C \in \mathcal{L}(X, Y)$ and $D \in \mathcal{L}(U, Y)$. In this context, it can be shown that the characterization (1.4) of conservativity is still valid. We must have $\mathcal{D}\left(A^{*}\right)=\mathcal{D}(A)$ and the first equation in (1.4) holds on $\mathcal{D}(A)$. The proof is not difficult and it will be given in Section 4 (after Cor. 4.4). The property (1.5) is no longer true at this level of generality (however, it holds if the semigroups $\mathbb{T}$ and $\mathbb{T}^{*}$ are both strongly stable).

In general, leaving bounded $B$ and $C$ behind, the characterization of conservative well-posed linear systems is a more difficult problem, and it will be discussed elsewhere (see the comments in [27]). For extensions to nonlinear infinite-dimensional systems see Ball [2] and Maschke and van der Schaft [12]. 
This paper is about a special class of conservative linear systems, which are described by a second order differential equation (in a Hilbert space) and an output equation. The equations are simple and occur often as models of physical systems. Well-posedness is not assumed a priori: it is proved, together with conservativity. The operators $B$ and $C$ are not assumed to be bounded, so that we cannot use the characterization (1.4) of conservativity.

We outline our construction and state the main results. The proofs will be provided in the later sections. Let $H$ be a Hilbert space, and let $A_{0}: \mathcal{D}\left(A_{0}\right) \rightarrow H$ be a self-adjoint, positive and boundedly invertible operator. We introduce the scale of Hilbert spaces $H_{\alpha}, \alpha \in \mathbb{R}$, as follows: for every $\alpha \geq 0, H_{\alpha}=\mathcal{D}\left(A_{0}^{\alpha}\right)$, with the norm $\|z\|_{\alpha}=\left\|A_{0}^{\alpha} z\right\|_{H}$. The space $H_{-\alpha}$ is defined by duality with respect to the pivot space $H$ as follows: $H_{-\alpha}=H_{\alpha}^{*}$ for $\alpha>0$. Equivalently, $H_{-\alpha}$ is the completion of $H$ with respect to the norm $\|z\|_{-\alpha}=\left\|A_{0}^{-\alpha} z\right\|_{H}$. The operator $A_{0}$ can be extended (or restricted) to each $H_{\alpha}$, such that it becomes a bounded operator

$$
A_{0}: H_{\alpha} \rightarrow H_{\alpha-1} \quad \forall \alpha \in \mathbb{R}
$$

The second ingredient needed for our construction is a bounded linear operator $C_{0}: H_{\frac{1}{2}} \rightarrow U$, where $U$ is another Hilbert space. We identify $U$ with its dual, so that $U=U^{*}$. We denote $B_{0}=C_{0}^{*}$, so that $B_{0}: U \rightarrow H_{-\frac{1}{2}}$.

The aim of this work is the study of the system described by

$$
\begin{gathered}
\frac{\mathrm{d}^{2}}{\mathrm{~d} t^{2}} z(t)+A_{0} z(t)+\frac{1}{2} B_{0} \frac{\mathrm{d}}{\mathrm{d} t} C_{0} z(t)=B_{0} u(t), \\
y(t)=-\frac{\mathrm{d}}{\mathrm{d} t} C_{0} z(t)+u(t),
\end{gathered}
$$

where $t \in[0, \infty)$ is the time. The equation (1.7) is understood as an equation in $H_{-\frac{1}{2}}$, i.e., all the terms are in $H_{-\frac{1}{2}}$. Most of the linear equations modelling the damped vibrations of elastic structures can be written in the form (1.7), where $z$ stands for the displacement field and the term $B_{0} \frac{\mathrm{d}}{\mathrm{d} t} C_{0} z(t)$, informally written as $B_{0} C_{0} \dot{z}(t)$, represents a viscous feedback damping. The signal $u(t)$ is an external input with values in $U$ (often a displacement, a force or a moment acting on the boundary) and the signal $y(t)$ is the output (measurement) with values in $U$ as well. The state $x(t)$ of this system and its state space $X$ are defined by

$$
x(t)=\left[\begin{array}{c}
z(t) \\
\dot{z}(t)
\end{array}\right], \quad X=H_{\frac{1}{2}} \times H .
$$

This means that in order to solve (1.7), initial values for $z(t)$ and $\dot{z}(t)$ at $t=0$ have to be specified, and we take $z(0) \in H_{\frac{1}{2}}$ and $\dot{z}(0) \in H$. As we shall see, if $u \in L^{2}([0, \infty), U)$ then also $y \in L^{2}([0, \infty), U)$.

We need some notation: for any Hilbert space $W$, the Sobolev spaces $\mathcal{H}^{p}(0, \infty ; W)$ of $W$-valued functions (with $p \in \mathbb{N}$ ) are defined in the usual way, see [11]. The larger spaces $\mathcal{H}_{\text {loc }}^{p}(0, \infty ; W)$ (with $\left.p \in \mathbb{N}\right)$ are defined recursively: $\mathcal{H}_{\mathrm{loc}}^{0}(0, \infty ; W)=L_{\mathrm{loc}}^{2}([0, \infty), W)$ and for $p \in \mathbb{N}, f \in \mathcal{H}_{\mathrm{loc}}^{p}(0, \infty ; W)$ if $f$ is continuous and

$$
f(\tau)-f(0)=\int_{0}^{\tau} v(t) \mathrm{d} t \quad \forall \tau \geq 0,
$$

for some $v \in \mathcal{H}_{\mathrm{loc}}^{p-1}(0, \infty ; W)$. The notation $C^{n}(0, \infty ; W)$ (with $\left.n \in\{0,1,2, \ldots\}\right)$ for $n$ times continuously differentiable $W$-valued functions on $[0, \infty)$ is also quite standard (at $t=0$ we consider the derivative from the right, of course). We denote by $B C^{n}(0, \infty ; W)$ the space of those $f \in C^{n}(0, \infty ; W)$ for which $f, f^{\prime}, \ldots f^{(n)}$ are 
all bounded on $[0, \infty)$. We write $C$ instead of $C^{0}$. Our main result is the following:

Theorem 1.1. With $U, H, A_{0}, H_{\alpha}, C_{0}, B_{0}$ and $X$ as above, the equations (1.7) and (1.8) determine a conservative linear system $\Sigma$, in the following sense:

There exists a conservative linear system $\Sigma$ whose input and output spaces are both $U$, whose state space is $X$ and which has the following properties: if $u \in L^{2}([0, \infty), U)$ is the input function, $x_{0}=\left[\begin{array}{l}z_{0} \\ w_{0}\end{array}\right] \in X$ is the initial state, $x=\left[\begin{array}{c}z \\ w\end{array}\right]$ is the corresponding state trajectory and $y$ is the corresponding output function, then

(1)

$$
z \in B C\left(0, \infty ; H_{\frac{1}{2}}\right) \cap B C^{1}(0, \infty ; H) \cap \mathcal{H}_{\mathrm{loc}}^{2}\left(0, \infty ; H_{-\frac{1}{2}}\right) ;
$$

(2) the two components of $x$ are related by $w=\dot{z}$;

(3) $C_{0} z \in \mathcal{H}^{1}(0, \infty ; U)$ and the equations (1.7) (in $H_{-\frac{1}{2}}$ ) and (1.8) (in $U$ ) hold for almost every $t \geq 0$ (hence, $y \in L^{2}([0, \infty), U)$ ).

Note that the property $C_{0} z \in \mathcal{H}^{1}(0, \infty ; U)$ (contained in point (3) of the theorem) implies that $\lim _{t \rightarrow \infty} C_{0} z(t)$ $=0$ (for any initial state and any $L^{2}$ input function). This is remarkable, because the system $\Sigma$ is not strongly stable in general.

If we make additional smoothness assumptions on the input signal $u$ and the initial conditions $z_{0}$ and $w_{0}$, as well as a compatibility assumption, then we get smoother state trajectories and output functions. Then, the equations (1.7) and (1.8) can be rewritten in a somewhat simpler form, as the following theorem shows:

Theorem 1.2. With the assumptions and the notation of Theorem 1.1, introduce the Hilbert the space $Z_{0}=$ $H_{1}+A_{0}^{-1} B_{0} U \subset H_{\frac{1}{2}}$, with the norm

$$
\|z\|_{Z_{0}}^{2}=\inf \left\{\begin{array}{l|l}
\left\|z_{1}\right\|_{1}^{2}+\|v\|^{2} & \begin{array}{l}
z=z_{1}-A_{0}^{-1} B_{0} v \\
z_{1} \in H_{1}, \quad v \in U
\end{array}
\end{array}\right\}
$$

Suppose that $u \in \mathcal{H}^{1}(0, \infty ; U), z_{0}, w_{0} \in H_{\frac{1}{2}}$ and

$$
A_{0} z_{0}+\frac{1}{2} B_{0} C_{0} w_{0}-B_{0} u(0) \in H
$$

(this implies $z_{0} \in Z_{0}$ ). If we denote by $z$ the solution of (1.7) with $z(0)=z_{0}$ and $\dot{z}(0)=w_{0}$, and if we denote by $y$ the output defind by (1.8), then

$$
z \in B C\left(0, \infty ; Z_{0}\right) \cap B C^{1}\left(0, \infty ; H_{\frac{1}{2}}\right) \cap B C^{2}(0, \infty ; H),
$$

$y \in \mathcal{H}^{1}(0, \infty ; U)$ and we have for every $t \geq 0$

$$
\begin{gathered}
\ddot{z}(t)+A_{0} z(t)+\frac{1}{2} B_{0} C_{0} \dot{z}(t)=B_{0} u(t), \\
y(t)=-C_{0} \dot{z}(t)+u(t) .
\end{gathered}
$$

It is easy to verify that the equations $(1.11,1.12)$ are equivalent to the following system of first order equations:

$$
\left\{\begin{array}{l}
\dot{x}(t)=A x(t)+B u(t) \\
y(t)=\bar{C} x(t)+u(t)
\end{array}\right.
$$


where

$$
\begin{gathered}
A=\left[\begin{array}{cc}
0 & I \\
-A_{0} & -\frac{1}{2} B_{0} C_{0}
\end{array}\right], \quad B=\left[\begin{array}{c}
0 \\
B_{0}
\end{array}\right], \\
\mathcal{D}(A)=\left\{\left[\begin{array}{c}
z \\
w
\end{array}\right] \in H_{\frac{1}{2}} \times H_{\frac{1}{2}} \mid A_{0} z+\frac{1}{2} B_{0} C_{0} w \in H\right\}, \\
\bar{C}: Z_{0} \times H_{\frac{1}{2}} \rightarrow U, \quad \bar{C}=\left[\begin{array}{ll}
0 & -C_{0}
\end{array}\right] .
\end{gathered}
$$

We denote by $C$ the restriction of $\bar{C}$ to $\mathcal{D}(A)$. For the concepts of semigroup generator, control operator, observation operator and transfer function of a well-posed linear system, we refer again to [24,25], or to Section 3. We denote by $\mathbb{C}_{0}$ the open right half-plane in $\mathbb{C}$ (where $\operatorname{Re} s>0$ ).

Theorem 1.3. With the notation from Theorem 1.1 together with the above notation, the semigroup generator of $\Sigma$ is $A$, its control operator is $B$ and its observation operator is $C$. The transfer function of $\Sigma$ is given for all $s \in \mathbb{C}_{0}$ by

$$
\mathbf{G}(s)=\bar{C}(s I-A)^{-1} B+I=I-C_{0} s\left(s^{2} I+A_{0}+\frac{s}{2} B_{0} C_{0}\right)^{-1} B_{0}
$$

and we have $\|\mathbf{G}(s)\| \leq 1$ for all $s \in \mathbb{C}_{0}$. The function $\mathbf{G}$ has an analytic continuation to a neighborhood of 0 and, denoting $T=C_{0} A_{0}^{-1} B_{0}$, we have

$$
\mathbf{G}(0)=I, \quad \mathbf{G}^{\prime}(0)=-T, \quad \mathbf{G}^{\prime \prime}(0)=T^{2} .
$$

Note that the operator $T \in \mathcal{L}(U)$ introduced above is self-adjoint and $T \geq 0$. The formulas (1.15) show that the first three terms in the Taylor expansion of $\mathbf{G}$ around zero agree with the first three terms in the expansion of $\exp (-T s)$. Thus, for small $|s|, \mathbf{G}(s)$ can be approximated by $\exp (-T s)$. Unfortunately, the higher order terms in the two Taylor expansions do not agree in general (but they do agree for the one-dimensional wave equation, see the comments at the end of Sect. 7).

The last part of Theorem 1.3 shows that not every conservative system with equal input and output spaces is isomorphic to a system of the type discussed in Theorem 1.1. Indeed, for conservative systems in general, G may be any analytic function on $\mathbb{C}_{0}$ whose values are contractions, see [1] (pp. 32-33), and such transfer functions do not have to satisfy $\mathbf{G}(0)=I$ or $\mathbf{G}^{\prime \prime}(0)=\left[\mathbf{G}^{\prime}(0)\right]^{2}$.

The following theorem refers to a special subclass of the systems treated in the first three theorems. If the system $\Sigma$ originates from a partial differential equation with boundary control, then it usually belongs to this subclass. The theorem is related to the theory of abstract boundary control systems in Salamon [15].

Theorem 1.4. With the assumptions and the notation of Theorem 1.1, and with the Hilbert space $Z_{0}$ defined as in Theorem 1.2, suppose that there exists an operator $G_{0} \in \mathcal{L}\left(Z_{0}, U\right)$ such that

$$
G_{0} H_{1}=\{0\}, \quad G_{0} A_{0}^{-1} B_{0}=I .
$$

For every $z \in Z_{0}$, we define $L_{0} z=A_{0} z-B_{0} G_{0} z$ (here we have used the extension of $A_{0}$ to $H_{\frac{1}{2}}$, as in (1.6)). Then $\operatorname{Ker} G_{0}=H_{1}, \quad L_{0} \in \mathcal{L}\left(Z_{0}, H\right)$ and the system $\Sigma$ can be described by the equations

$$
\left\{\begin{aligned}
\ddot{z}(t)+L_{0} z(t) & =0, \\
G_{0} z(t)+\frac{1}{2} C_{0} \dot{z}(t) & =u(t), \\
G_{0} z(t)-\frac{1}{2} C_{0} \dot{z}(t) & =y(t),
\end{aligned}\right.
$$


in the following sense:

(1) if $u \in \mathcal{H}^{1}(0, \infty ; U), z_{0} \in Z_{0}$ and $w_{0} \in H_{\frac{1}{2}}$, then the condition

$$
G_{0} z_{0}+\frac{1}{2} C_{0} w_{0}=u(0)
$$

is equivalent to (1.9). Hence, if the above condition holds, if we denote by $z$ the solution of (1.7) with $z(0)=z_{0}$ and $\dot{z}(0)=w_{0}$, and if we denote by $y$ the output defined by (1.8), then (by Th. 1.2) (1.10) holds and $y \in \mathcal{H}^{1}(0, \infty ; U)$;

(2) the equations (1.16) are equivalent to (1.11) and (1.12) (and hence also to (1.13)). This means that $z \in C\left(0, \infty ; Z_{0}\right) \cap C^{1}\left(0, \infty ; H_{\frac{1}{2}}\right) \cap C^{2}(0, \infty ; H)$ together with $u, y \in C(0, \infty ; U)$ satisfy $(1.16)$ if and only if they satisfy (1.11) and (1.12).

Corollary 1.5. With the assumptions and the notation of Theorem 1.4, if $u, z$ and $y$ are as in statement (1) of the theorem, then for every $t \geq 0$

$$
\frac{\mathrm{d}}{\mathrm{d} t}\left\|\left[\begin{array}{c}
z(t) \\
\dot{z}(t)
\end{array}\right]\right\|_{X}^{2}=\|u(t)\|_{U}^{2}-\|y(t)\|_{U}^{2}=2 \operatorname{Re}\left\langle G_{0} z(t), C_{0} \dot{z}(t)\right\rangle .
$$

The proof of the results stated above, together with other intermediate or related results, will be given in Sections 4-6. Sections 2, 3 are dedicated to the background on infinite-dimensional systems. In Section 7 we describe a wave equation on an $n$-dimensional domain which fits into the framework of this paper.

For conservative systems of the type discussed in this paper, there is a rich structure linking the various controllability, observability and stability properties. This structure will be explored in Part II of this paper, which will also contain further relevant examples described by partial differential equations.

\section{Admissible CONTROL AND OBSERVATION OPERATORS}

In this section we recall the terminology and some results on admissibility (in particular, infinite-time admissibility) following $[22,23]$ and [8]. These concepts and results are due to a large number of researchers, and we refer to $[3,5,8,9,22,23]$ for further references and historical remarks.

Throughout this section, $X$ is a Hilbert space and $A: \mathcal{D}(A) \rightarrow X$ is the generator of a strongly continuous semigroup $\mathbb{T}$ on $X$. The Hilbert space $X_{1}$ is $\mathcal{D}(A)$ with the norm $\|z\|_{1}=\|(\beta I-A) z\|$, where $\beta \in \rho(A)$ is fixed (this norm is equivalent to the graph norm). The Hilbert space $X_{-1}$ is the completion of $X$ with respect to the norm $\|z\|_{-1}=\left\|(\beta I-A)^{-1} z\right\|$. This space is isomorphic to $\mathcal{D}\left(A^{*}\right)^{*}$, and we have

$$
X_{1} \subset X \subset X_{-1}
$$

densely and with continuous embeddings. $\mathbb{T}$ extends to a semigroup on $X_{-1}$, denoted by the same symbol. The generator of this extended semigroup is an extension of $A$, whose domain is $X$, so that $A: X \rightarrow X_{-1}$. This extension of $A$ will be denoted by $A$ as well, so that we have $(s I-A)^{-1} \in \mathcal{L}\left(X_{-1}, X\right)$.

Suppose that $U$ is a Hilbert space and $B \in \mathcal{L}\left(U, X_{-1}\right) . \quad B$ is an admissible control operator for $\mathbb{T}$ if the following property holds: if $x$ is the solution of

$$
\dot{x}(t)=A x(t)+B u(t)
$$

with $x(0)=x_{0} \in X$ and $u \in L^{2}([0, \infty), U)$, then $x(t) \in X$ for all $t \geq 0$. In this case, $x$ is a continuous $X$-valued function of $t$ and

$$
x(t)=\mathbb{T}_{t} x_{0}+\Phi_{t} u,
$$


where $\Phi_{t} \in \mathcal{L}\left(L^{2}([0, \infty), U), X\right)$ is defined by

$$
\Phi_{t} u=\int_{0}^{t} \mathbb{T}_{t-\sigma} B u(\sigma) \mathrm{d} \sigma
$$

The above integration is done in $X_{-1}$, but the result is in $X$. The admissibility of $B$ is equivalent to the fact that for some (hence, for every) $t>0$, the range of $\Phi_{t}$ is in $X$. The Laplace transform of $x$ from $(2.2)$ is

$$
\hat{x}(s)=(s I-A)^{-1}\left[x_{0}+B \hat{u}(s)\right] .
$$

In this context, $B$ is called bounded if $B \in \mathcal{L}(U, X)$ (and unbounded otherwise). We have $\Phi_{\tau} \mathbf{P}_{\tau}=\Phi_{\tau}$ (causality), so that $\Phi_{\tau}$ has an obvious extension to $L_{\text {loc }}^{2}([0, \infty), U)$, which will be used frequently in the next sections.

The admissible control operator $B$ is called infinite-time admissible if the state trajectory $x$ from (2.2) corresponding to $x_{0}=0$ is bounded, for any $u \in L^{2}([0, \infty), U)$. This is equivalent to the statement that the operators $\Phi_{t}$ are uniformly bounded. An equivalent formulation of infinite-time admissibility is as follows: $B \in \mathcal{L}\left(U, X_{-1}\right)$ is an infinite-time admissible control operator for $\mathbb{T}$ if and only if, for every $u \in L^{2}([0, \infty), U)$, the following limit exists in $X$ :

$$
\tilde{\Phi} u=\lim _{\tau \rightarrow \infty} \int_{0}^{\tau} \mathbb{T}_{t} B u(t) \mathrm{d} t
$$

(this does not mean that we can integrate from 0 to $\infty$ ). If $\mathbb{T}$ is exponentially stable and $B$ is an admissible control operator, then $B$ is infinite-time admissible.

Suppose that $Y$ is a Hilbert space and $C \in \mathcal{L}\left(X_{1}, Y\right)$. C is an admissible observation operator for $\mathbb{T}$ if for every $T>0$ there exists a $K_{T} \geq 0$ such that

$$
\int_{0}^{T}\left\|C \mathbb{T}_{t} x_{0}\right\|^{2} \mathrm{~d} t \leq K_{T}^{2}\left\|x_{0}\right\|^{2} \quad \forall x_{0} \in \mathcal{D}(A) .
$$

In this context, $C$ is called bounded if it can be extended such that $C \in \mathcal{L}(X, Y)$ (and it is called unbounded otherwise).

We regard $L_{\text {loc }}^{2}([0, \infty), Y)$ as a Fréchet space with the seminorms being the $L^{2}$ norms on the intervals $[0, n], n \in \mathbb{N}$. Then the admissibility of $C$ means that there is a continuous operator $\Psi: X \rightarrow L_{\text {loc }}^{2}([0, \infty), Y)$ such that

$$
\left(\Psi x_{0}\right)(t)=C \mathbb{T}_{t} x_{0} \quad \forall x_{0} \in \mathcal{D}(A) .
$$

The operator $\Psi$ is completely determined by (2.7), because $\mathcal{D}(A)$ is dense in $X$. If $x_{0} \in X$ and $y=\Psi x_{0}$, then its Laplace transform is

$$
\hat{y}(s)=C(s I-A)^{-1} x_{0} .
$$

The admissible observation operator $C$ is called infinite-time admissible if the range of $\Psi$ is in $L^{2}([0, \infty), Y)($ and then it follows that $\left.\Psi \in \mathcal{L}\left(X, L^{2}([0, \infty), Y)\right)\right)$. If $\mathbb{T}$ is exponentially stable and $C$ is an admissible observation operator for $\mathbb{T}$, then $C$ is infinite-time admissible. The following duality result holds: $C$ is an (infinite-time) admissible observation operator for $\mathbb{T}$ if and only if $C^{*}$ is an (infinite-time) admissible control operator for the adjoint semigroup $\mathbb{T}^{*}$.

In the sequel, for any Hilbert space $W$, any $\tau>0$ and any integer $m \geq 0$, we denote by $\mathcal{H}^{m}(0, \tau ; W)$ the space of all the functions $h \in L^{2}([0, \tau], W)$ for which all the derivatives $h^{(k)}$ (in the sense of distributions), with $k \leq m$, belong to $L^{2}([0, \tau], W)$.

Assume again that $U$ is a Hilbert space and $B \in \mathcal{L}\left(U, X_{-1}\right)$ ( $B$ is not assumed to be admissible). The operators $\Phi_{t}$ are defined as in $(2.3)$ and they map $L^{2}([0, \infty), U)$ into $X_{-1}$. We introduce the space $Z$ by

$$
Z=X_{1}+(\beta I-A)^{-1} B U
$$


This is a Hilbert space if we define on it the norm by

$$
\|z\|_{Z}^{2}=\inf \left\{\begin{array}{l|l}
\left\|z_{1}\right\|_{1}^{2}+\|v\|^{2} & \begin{array}{c}
z=z_{1}+(\beta I-A)^{-1} B v \\
z_{1} \in X_{1}, \quad v \in U
\end{array}
\end{array}\right\}
$$

(Thus, $Z$ is isomorphic to a closed subspace of $X_{1} \times U$, namely to the orthogonal complement of those $\left(z_{1}, v\right)$ for which $z_{1}+(\beta I-A)^{-1} B v=0$.) We have $X_{1} \subset Z \subset X$, with continuous embeddings, and $(\beta I-A)^{-1} B \in \mathcal{L}(U, Z)$ for every $\beta \in \rho(A)$. The following result appears without proof in Salamon [15] (as part of his Lem. 2.3). The proof is not difficult and it is omitted.

Proposition 2.1. With $X, \mathbb{T}, A, X_{1}, X_{-1}, U, B, Z$ and $\Phi_{t}$ as introduced above, let the function $x:[0, \tau] \rightarrow X_{-1}$ be defined by (2.2), where

$$
u \in \mathcal{H}^{2}(0, \tau ; U) \quad \text { and } \quad A x_{0}+B u(0) \in X .
$$

Then $x \in C([0, \tau], Z) \cap C^{1}([0, \tau], X)$ and $\dot{x}(t)=A x(t)+B u(t)$ holds in $X$.

If $B$ is admissible for $\mathbb{T}$, then the above properties remain true for all $u$ in the larger space $\mathcal{H}^{1}(0, \tau ; U)$, if again $A x_{0}+B u(0) \in X$.

\section{BACKGROUND ON WELL-POSED LINEAR SYSTEMS}

In this section we recall briefly some well known facts about well-posed linear systems, their transfer functions and regular linear systems, as well as some relatively new material on the combined observation/feedthrough operator. Proposition 3.1 (which will be needed in Sect. 6) is new. For the other results we do not give proofs, but we indicate the relevant references.

A well-posed linear system is a linear time-invariant system such that on any finite time interval, the operator from the initial state and the input function to the final state and the output function is bounded. To express this more clearly, let us denote by $U$ the input space, by $X$ the state space and by $Y$ the output space of a well-posed linear system $\Sigma$. $U, X$ and $Y$ are complex Hilbert spaces and the input and output functions are $u \in L_{\text {loc }}^{2}([0, \infty), U)$ and $y \in L_{\text {loc }}^{2}([0, \infty), Y)$. The state trajectory $x$ is an $X$-valued function. These functions are related by (1.1), for all $\tau \geq 0$. The boundedness property mentioned earlier means that the operators $\Sigma_{\tau}$ are bounded. Denoting $c_{\tau}=\left\|\Sigma_{\tau}\right\|$, this can be written as

$$
\|x(\tau)\|^{2}+\int_{0}^{\tau}\|y(t)\|^{2} \mathrm{~d} t \leq c_{\tau}^{2}\left(\|x(0)\|^{2}+\int_{0}^{\tau}\|u(t)\|^{2} \mathrm{~d} t\right) .
$$

The operators $\Sigma_{\tau}$ are partitioned in a natural way (corresponding to the two product spaces) as follows:

$$
\Sigma_{\tau}=\left[\begin{array}{ll}
\mathbb{T}_{\tau} & \Phi_{\tau} \\
\Psi_{\tau} & \mathbb{F}_{\tau}
\end{array}\right]
$$

The four families of operators appearing on the right-hand side above must satisfy four functional equations expressing the causality and the time-invariance of $\Sigma$ (these functional equations are parts of the definition of a well-posed system). For the details we refer to Salamon [15,16] or Staffans [17] or Weiss [24,25]. These papers (and also others) offer equivalent definitions but formulated quite differently, so that their equivalence is not obvious. In the formalism of $[24,25]$, which we follow here, $\Sigma$ is defined to be the family of all $\Sigma_{\tau}$, i.e., $\Sigma=\left(\Sigma_{\tau}\right)_{\tau \geq 0}$. We shall now state facts about well-posed linear systems, with the following convention: unless a different reference is given, the fact can be found in [24] or [25].

Let $\Sigma$ be a well-posed linear system with input space $U$, state space $X$ and output space $Y$. Let $\mathbb{T}$ be the semigroup of $\Sigma$, i.e., the strongly continuous semigroup on $X$ which describes the evolution of the state of $\Sigma$ if the input function is zero: $x(t)=\mathbb{T}_{t} x(0)$. Let $A$ denote the generator of $\mathbb{T}$. The Hilbert spaces $X_{1}$ and $X_{-1}$ are defined like at the beginning of Section 2. There exists a unique operator $B \in \mathcal{L}\left(U, X_{-1}\right)$, called the control operator of $\Sigma$, with the following property: let $x_{0} \in X$ denote the initial state of $\Sigma$ and let $u \in L_{\mathrm{loc}}^{2}([0, \infty), U)$ 
be its input function. Then the state of $\Sigma$ at any moment $\tau \geq 0$ can be expressed by the formula $(2.2)$, where $\Phi_{\tau}$ is defined as in (2.3). We have $x(\tau) \in X$ (that is, $B$ is admissible) and $x(\tau)$ depends continuously on $\tau$, on $x_{0}$ and on $\mathbf{P}_{\tau} u$ (u restricted to $\left.[0, \tau]\right)$. The state trajectory $x$ is the unique strong solution of $(2.1)$ in $X_{-1}$, with the given $x_{0}$ and $u$. The operators $\mathbb{T}_{\tau}$ and $\Phi_{\tau}$ appear in the upper row of $\Sigma_{\tau}$ in (3.2).

There exists a unique operator $C \in \mathcal{L}\left(X_{1}, Y\right)$, called the observation operator of $\Sigma$, with the following property: if the input function is $u=0$ and the initial state $x_{0}$ is in $X_{1}$, then the output function $y$ of $\Sigma$ is

$$
y(t)=C \mathbb{T}_{t} x_{0}, \quad \forall t \geq 0 .
$$

Since (by the definition of a well-posed system) we have that for all $\tau \geq 0, \mathbf{P}_{\tau} y \in L^{2}([0, \tau], Y)$ depends continuously on $x_{0} \in X$, it is clear that $C$ must be admissible. Let $\Psi$ be the operator defined in (2.7) (with continuous extension to $X$ ), so that

$$
\Psi: X \rightarrow L_{\mathrm{loc}}^{2}([0, \infty), Y)
$$

continuously (remember that $L_{\text {loc }}^{2}([0, \infty), Y)$ is a Fréchet space with the seminorms $\left.\|y\|_{n}=\left\|\mathbf{P}_{n} y\right\|_{L^{2}}\right)$. $\Psi$ is called the extended output map of $\Sigma$. The operators $\Psi_{\tau}=\mathbf{P}_{\tau} \Psi$ are in $\mathcal{L}\left(X, L^{2}([0, \tau], Y)\right)$ and they occupy the left lower corner of $\Sigma_{\tau}$ in $(3.2)$. We regard $L^{2}([0, \tau], Y)$ as a subspace of $L^{2}([0, \infty), Y)$ (which in turn is a subspace of $\left.L_{\text {loc }}^{2}([0, \infty), Y)\right)$ by extending functions to be zero outside $[0, \tau]$. Thus, $\Psi_{\tau}$ may also be regarded as an operator from $X$ to $L^{2}([0, \infty), Y)$, as it is usually done.

If the initial state is $x_{0}=0$, then the output function $y$ depends only on the input function $u: y=\mathbb{F} u$. The continuous linear operator

$$
\mathbb{F}: L_{\mathrm{loc}}^{2}([0, \infty), U) \rightarrow L_{\mathrm{loc}}^{2}([0, \infty), Y)
$$

is called the extended input-output map of $\Sigma$. We have $\mathbf{P}_{\tau} \mathbb{F}=\mathbf{P}_{\tau} \mathbb{F} \mathbf{P}_{\tau}$ (causality) and $\mathbb{F}$ commutes with the right-shift operators on the appropriate spaces (time-invariance). The operators $\mathbb{F}_{\tau}=\mathbf{P}_{\tau} \mathbb{F}$ (with $\tau \geq 0$ ) are bounded from $L^{2}([0, \infty), U)$ to $L^{2}([0, \tau], Y)$ and they occupy the right lower corner of $\Sigma_{\tau}$ in (3.2). Similarly as for $\Psi_{\tau}, \mathbb{F}_{\tau}$ may also be regarded as being in $\mathcal{L}\left(L^{2}([0, \infty), U), L^{2}([0, \infty), Y)\right)$.

The operator $\mathbb{F}$ can be described by its transfer function (also called the transfer function of $\Sigma$ ), which is an analytic $\mathcal{L}(U, Y)$-valued function $\mathbf{G}$ defined on some right half-plane $\mathbb{C}_{\mu}=\{s \in \mathbb{C} \mid \operatorname{Re} s>\mu\}$. Denoting the growth bound of $\mathbb{T}$ by $\omega_{0}$, the domain of $\mathbf{G}$ includes $\mathbb{C}_{\omega_{0}}$. If $\omega>\omega_{0}$, the function $\mathrm{e}^{-\omega t} u(t)$ is in $L^{2}([0, \infty), U)$ and if $y=\mathbb{F} u$, then the function $\mathrm{e}^{-\omega t} y(t)$ is in $L^{2}([0, \infty), Y)$ and $\hat{y}(s)=\mathbf{G}(s) \hat{u}(s)$ holds for $s \in \mathbb{C}_{\omega}$. The transfer function of any well-posed linear system is well-posed, meaning that it is bounded on some right half-plane.

The operator $\mathbb{F}$ is bounded from $L^{2}([0, \infty), U)$ to $L^{2}([0, \infty), Y)$ if and only if $\mathbf{G}$ is bounded on $\mathbb{C}_{0}$. If this is the case, then

$$
\|\mathbb{F}\|_{\mathcal{L}\left(L^{2}\right)}=\sup _{\operatorname{Re} s>0}\|\mathbf{G}(s)\| .
$$

If $\mathbf{G}$ is the transfer function of $\Sigma$, then its derivative is

$$
\mathbf{G}^{\prime}(s)=-C(s I-A)^{-2} B
$$

for all $s \in \mathbb{C}_{\omega_{0}}$, where $\omega_{0}$ is the growth bound of $\mathbb{T}$. Hence, $\mathbf{G}$ is determined by $A, B$ and $C$ up to an additive constant operator.

The operator $C$ has at least one extension $\bar{C} \in \mathcal{L}(Z, Y)$, where $Z$ is the space from (2.9) and (2.10), see Theorem 3.4 in [19]. For any such $\bar{C}$, denoting

$$
D=\mathbf{G}(\beta)-\bar{C}(\beta I-A)^{-1} B
$$

where $\beta \in \mathbb{C}_{\omega_{0}}$, we have that $D$ is independent of the choice of $\beta$. Using $\bar{C}$ and $D$, we give a description of the output function of $\Sigma$ in the time domain, which is valid under additional assumptions on the input function and the initial state (we follow Sect. 3 of [19]). Take $u \in \mathcal{H}^{1}(0, \infty ; U)$ and $x_{0} \in X$ such that the compatibility condition $A x_{0}+B u(0) \in X$ holds. Let $x$ be the corresponding state trajectory given by $(2.2)$ and let $y$ be the corresponding output function, i.e.,

$$
y=\Psi x_{0}+\mathbb{F} u .
$$


It follows from Proposition 2.1 that for every $t \geq 0, \dot{x}(t) \in X, x(t) \in Z$ and

$$
\dot{x}(t)=A x(t)+B u(t) \quad \forall t \geq 0 .
$$

For any $\omega>\omega_{0}$, the function $\mathrm{e}^{-\omega t} y(t)$ is in $\mathcal{H}^{1}(0, \infty ; Y)$ and

$$
y(t)=\bar{C} x(t)+D u(t) \quad \forall t \geq 0 .
$$

We will need the following proposition, giving sufficient conditions for a quadruple of operators $(A, B, \bar{C}, D)$ to define a well-posed system via (3.7) and (3.8). These equations will hold for all pairs $\left(x_{0}, u\right)$ that are as in the text leading to (3.6) (and these pairs are dense in $X \times L^{2}([0, \infty), U)$ ). We need the notation

$$
\begin{gathered}
\mathcal{H}_{0}^{1}(0, \infty ; U)=\left\{u \in \mathcal{H}^{1}(0, \infty ; U) \mid u(0)=0\right\}, \\
\mathcal{H}_{L}^{2}(0, \infty ; U)=\mathcal{H}^{2}(0, \infty ; U) \cap \mathcal{H}_{0}^{1}(0, \infty ; U) .
\end{gathered}
$$

Proposition 3.1. Let $U, X$ and $Y$ be Hilbert spaces, let $A$ be the generator of a strongly continuous semigroup $\mathbb{T}$ on $X$ and let the spaces $X_{1}, X_{-1}$ be as in Section 2. Let $B \in \mathcal{L}\left(U, X_{-1}\right)$ be an admissible control operator for $\mathbb{T}$ and, for every $t \geq 0$, let $\Phi_{t}$ be the operator from (2.3). We define $Z$ as in (2.9) and (2.10). Assume that

$$
\bar{C} \in \mathcal{L}(Z, Y), \quad D \in \mathcal{L}(U, Y)
$$

are such that the restriction of $\bar{C}$ to $X_{1}$, denoted by $C$, is an admissible observation operator for $\mathbb{T}$. We define $\Psi$ as in (2.7) (with continuous extension to $X$ ) and for every $\tau \geq 0$ we put $\Psi_{\tau}=\mathbf{P}_{\tau} \Psi$, so that $\Psi_{\tau}$ $\in \mathcal{L}\left(X, L^{2}([0, \infty), Y)\right)$. We define

$$
\mathbb{F}: \mathcal{H}_{L}^{2}(0, \infty ; U) \rightarrow C(0, \infty ; Y)
$$

as follows: if $y=\mathbb{F} u$, then

$$
y(t)=\bar{C} \Phi_{t} u+D u(t) \quad \forall t \geq 0 .
$$

Assume that there exists $\tau>0$ and $k \geq 0$ such that for every $u$ and $y$ as above,

$$
\int_{0}^{\tau}\|y(t)\|^{2} \mathrm{~d} t \leq k^{2} \int_{0}^{\tau}\|u(t)\|^{2} \mathrm{~d} t .
$$

Then the last estimate remains valid for every $\tau \geq 0$ (with $k$ possibly depending on $\tau$ ), so that $\mathbb{F}$ has a unique continuous extension to an operator from $L_{\mathrm{loc}}^{2}([0, \infty), U)$ to $L_{\mathrm{loc}}^{2}([0, \infty), Y)$. Hence, if we denote $\mathbb{F}_{\tau}=\mathbf{P}_{\tau} \mathbb{F}$, then $\mathbb{F}_{\tau}$ is a bounded operator from $L^{2}([0, \infty), U)$ to $L^{2}([0, \infty), Y)$. Thus, we have defined the operators $\mathbb{T}_{\tau}, \Phi_{\tau}, \Psi_{\tau}$ and $\mathbb{F}_{\tau}$ for all $\tau \geq 0$. Now the operators $\Sigma_{\tau}$ from (3.2) define a well-posed linear system $\Sigma$ with input space $U$, state space $X$ and output space $Y$.

The semigroup generator of $\Sigma$ is A, its control operator is $B$ and its observation operator is $C$. The transfer function of $\Sigma$ is given, for all $s \in \mathbb{C}_{\omega_{0}}$ (where $\omega_{0}$ is the growth bound of $\mathbb{T}$ ) by

$$
\mathbf{G}(s)=\bar{C}(s I-A)^{-1} B+D .
$$

If $u \in \mathcal{H}^{1}(0, \infty ; U), x_{0} \in X, A x_{0}+B u(0) \in X$ and if we define $x$ by (2.2) and $y$ by (3.6), then $x$ and $y$ satisfy (3.7) and (3.8) for every $t \geq 0$.

The space $\mathcal{H}_{L}^{2}$ appearing in the proposition could have been replaced by various other spaces, for example, by $\mathcal{H}_{0}^{1}(0, \infty ; U)$, using the same proof, but we have stated the proposition in the way that suits us best in Section 6 .

The proof of this proposition is not difficult for a person familiar with the material in [19] and, for the sake of brevity, it will be omitted. Related results on the well-posedness of abstract system equations were derived in Salamon [15] and in Curtain and Weiss [4] (in [4], instead of (3.11) we had a condition on G). 


\section{Conservative linear systems}

According to the definition of conservative linear systems in Section $1, \Sigma=\left(\Sigma_{\tau}\right)_{\tau \geq 0}$ is conservative if $\Sigma_{\tau}$ is unitary (from $X \times L^{2}([0, \tau], U)$ to $X \times L^{2}([0, \tau], Y)$ ), for every $\tau \geq 0$. Here we derive a characterization of conservativity in terms of two differential equations, involving also the dual system.

For every $\tau \geq 0$ and every Hilbert space $W$, we define the reflection operator $\boldsymbol{9}_{\tau}$ acting on $L^{2}([0, \tau], W)$ by

$$
\left(\mathbf{Я}_{\tau} u\right)(t)=u(\tau-t)
$$

This operator can be extended to $L_{\text {loc }}^{2}([0, \infty), W)$ by taking $\left(\boldsymbol{Я}_{\tau} u\right)(t)=0$ for $t \geq \tau$.

Definition 4.1. Let $\Sigma=\left(\Sigma_{\tau}\right)_{\tau \geq 0}$ be a well-posed linear system with input space $U$, state space $X$ and output space $Y$. We partition $\Sigma_{\tau}$ as in (3.2). The dual system of $\Sigma$ is the family $\Sigma^{d}=\left(\Sigma_{\tau}^{d}\right)_{\tau \geq 0}$ defined by

$$
\Sigma_{\tau}^{d}=\left[\begin{array}{ll}
\mathbb{T}_{\tau}^{d} & \Phi_{\tau}^{d} \\
\Psi_{\tau}^{d} & \mathbb{F}_{\tau}^{d}
\end{array}\right]=\left[\begin{array}{ll}
I & 0 \\
0 & \boldsymbol{Я}_{\tau}
\end{array}\right]\left[\begin{array}{ll}
\mathbb{T}_{\tau}^{*} & \Psi_{\tau}^{*} \\
\Phi_{\tau}^{*} & \mathbb{F}_{\tau}^{*}
\end{array}\right]\left[\begin{array}{ll}
I & 0 \\
0 & \boldsymbol{Я}_{\tau}
\end{array}\right]
$$

It is not difficult to verify the $\Sigma^{d}$ is a well-posed linear system. This fact, as well as the proof of the following proposition, can be found in Staffans and Weiss [20].

Proposition 4.2. With $\Sigma$ and $\Sigma^{d}$ as in Definition 4.1, denote the semigroup generator, control operator and observation operator of $\Sigma$ by $A, B$ and $C$. If we denote the corresponding operators for $\Sigma^{d}$ by $A^{d}, B^{d}$ and $C^{d}$, then

$$
A^{d}=A^{*}, \quad B^{d}=C^{*}, \quad C^{d}=B^{*} .
$$

Moreover, the transfer functions of $\Sigma$ and $\Sigma^{d}$ are related by $\mathbf{G}^{d}(s)=\mathbf{G}(\bar{s})^{*}$.

It is clear from (4.1) that $\left(\Sigma^{d}\right)^{d}=\Sigma$ and that $\Sigma^{d}$ is conservative if and only if $\Sigma$ is conservative. The following proposition is easy to prove, using (1.2).

Proposition 4.3. With the notation of Proposition 4.2, let $u \in \mathcal{H}^{1}(0, \infty ; U)$, let $x_{0} \in X$ be such that Ax $x_{0}+$ $B u(0) \in X$, let $x$ be the state trajectory of $\Sigma$ corresponding to the initial state $x_{0}$ and the input function $u$, as in (2.2), and let $y$ be the corresponding output function (which is continuous, see the text after (3.6)). If $\Sigma_{\tau}$ is isometric for all $\tau \geq 0$, then the function $\|x(t)\|^{2}$ is in $C^{1}[0, \infty)$ and

$$
\frac{\mathrm{d}}{\mathrm{d} t}\|x(t)\|^{2}=\|u(t)\|^{2}-\|y(t)\|^{2} \quad \forall t \geq 0
$$

Conversely, if the above formula holds for every choice of $u$ in a subspace $\mathcal{E}$ of $\mathcal{H}^{1}(0, \infty ; U)$ which is dense in $L^{2}([0, \infty), U)$ (such as $\mathcal{E}=\mathcal{H}^{2}(0, \infty ; U)$ ) and for every $x_{0}$ satisfying $A x_{0}+B u(0) \in X$, then $\Sigma_{\tau}$ is isometric for all $\tau \geq 0$.

Corollary 4.4. Suppose that $\Sigma$ is a well-posed linear system with the property (4.2) (for all $x_{0}$ and $u$ satisfying suitable assumptions, as in Prop. 4.3). Suppose that also the dual system $\Sigma^{d}$ has the property (4.2). Then $\Sigma$ is conservative.

Indeed, by the "converse" part of Proposition 4.3, if $\Sigma$ has the property (4.2), then $\Sigma_{\tau}$ is isometric for all $\tau \geq 0$. Similarly, if $\Sigma^{d}$ has the property (4.2), then $\Sigma_{\tau}^{d}$ is isometric for all $\tau \geq 0$. By (4.1), $\Sigma_{\tau}^{*}$ is unitarily equivalent to $\Sigma_{\tau}^{d}$, so that both $\Sigma_{\tau}$ and $\Sigma_{\tau}^{*}$ are isometric. This clearly implies that $\Sigma_{\tau}$ is unitary, i.e., $\Sigma$ is conservative.

For well-posed systems with bounded $B$ and $C$, we can derive the characterization (1.4) of conservativity from the last corollary. Indeed, both sides of (4.2) can be writte as quadratic forms in $x(t)$ and $u(t)$. Equating the corresponding terms, we get a part of (1.4). We get the remaining part from the dual version of (4.2). 
Proposition 4.5. Let $\Sigma$ be a well-posed linear system with input space $U$, state space $X$, output space $Y$, semigroup $\mathbb{T}$, control operator $B$, observation operator $C$ and transfer function $\mathbf{G}$. Let $\mathcal{E}$ be a subspace of $\mathcal{H}^{1}(0, \infty ; U)$ which is dense in $L^{2}([0, \infty), U)$ (such as $\mathcal{E}=H^{2}(0, \infty ; U)$ ). Suppose that for every $u \in \mathcal{E}$ and for every $x_{0} \in X$ such that $A x_{0}+B u(0) \in X$, denoting by $x$ and $y$ the state trajectory and the output function of $\Sigma$ corresponding to the initial state $x_{0}$ and the input function $u$ (as in (2.2) and (3.6)), we have

$$
\frac{\mathrm{d}}{\mathrm{d} t}\|x(t)\|^{2} \leq\|u(t)\|^{2}-\|y(t)\|^{2} \quad \forall t \geq 0
$$

Then the following statements are true:

(1) $\mathbb{T}$ is a semigroup of contractions;

(2) $B$ is infinite-time admissible;

(3) $C$ is infinite-time admissible;

(4) $\|\mathbf{G}(s)\| \leq 1$ for all $s \in \mathbb{C}_{0}$.

Systems which have the property (4.3) are called dissipative. For more details on such systems we refer to Staffans and Weiss [19] (Sect. 7). In particular, the above proposition is contained in [19] (Prop. 7.2). It is clear that conservative systems are dissipative, and the dual of a dissipative system is dissipative.

The following proposition applies to all well-posed systems which satisfy certain four assumptions. These four assumptions are immediate consequences of the four statements in the previous proposition. Hence, the following proposition applies to all dissipative (in particular, to all conservative) linear systems.

Proposition 4.6. Let $\Sigma$ be a well-posed linear system with input space $U$, state space $X$, output space $Y$, semigroup $\mathbb{T}$, semigroup generator $A$, control operator $B$, observation operator $C$ and transfer function $\mathbf{G}$. Let $Z$ be the space from (2.9) and (2.10), let $\bar{C} \in \mathcal{L}(Z, Y)$ be an extension of $C$ and let $D \in \mathcal{L}(U, Y)$ be given by (3.5). We make the following four assumptions on $\Sigma$ :

(1) $\mathbb{T}$ is uniformly bounded;

(2) $B$ is infinite-time admissible;

(3) $C$ is infinite-time admissible;

(4) $\mathbf{G}$ is uniformly bounded on $\mathbb{C}_{0}$.

Let $x_{0} \in X$ and $u \in \mathcal{H}^{1}(0, \infty ; U)$ be such that $A x_{0}+B u(0) \in X$. Let $x$ and $y$ be the state trajectory and the output function of $\Sigma$ corresponding to the initial state $x_{0}$ and the input function $u$ (as in (2.2) and (3.6)). Then

$$
x \in B C(0, \infty ; Z) \cap B C^{1}(0, \infty ; X), \quad y \in \mathcal{H}^{1}(0, \infty ; Y) .
$$

Proof. This proposition and its proof are closely related to the first part of [19] (Th. 3.1) (without being a consequence of it).

We denote $\mathcal{U}=L^{2}([0, \infty), U)$. For every $t \geq 0$ we define on $X \times \mathcal{U}$ the bounded operator $\mathcal{G}_{t}$ by

$$
\mathcal{G}_{t}=\left[\begin{array}{cc}
\mathbb{T}_{t} & \Phi_{t} \\
0 & \mathbf{S}_{t}^{*}
\end{array}\right]
$$

where $\mathbf{S}_{t}^{*}$ is the left shift by $t$ on $\mathcal{U}$. Then assumptions $(1)$ and $(2)$ imply that $\mathcal{G}=\left(\mathcal{G}_{t}\right)_{t \geq 0}$ is a uniformly bounded strongly continuous semigroup on $X \times \mathcal{U}$. We denote by $\mathcal{A}$ the generator of $\mathcal{G}$, and then we have $\mathcal{D}(\mathcal{A}) \subset Z \times \mathcal{H}^{1}(0, \infty ; U)$, with continuous embedding (a precise decription of $\mathcal{A}$ is given in [19]). The conditions imposed on $x_{0}$ and $u$ mean that $\xi_{0}=\left[\begin{array}{c}x_{0} \\ u\end{array}\right] \in \mathcal{D}(\mathcal{A})$. It follows that the function $\xi$ defined by $\xi(t)=\mathcal{G}_{t}\left[\begin{array}{c}x_{0} \\ u\end{array}\right]$ is in $B C^{1}(0, \infty ; X \times \mathcal{U})$, which implies that $x$ (the first component of $\xi$ ) is in $B C^{1}(0, \infty ; X)$, as claimed. The fact that $\xi_{0} \in \mathcal{D}(\mathcal{A})$ also implies that $\xi$ is bounded and continuous with values in $\mathcal{D}(\mathcal{A})$ (with the graph norm), which implies that $x$ (the first component of $\xi$ ) is in $B C(0, \infty ; Z)$, as claimed. 
To prove our claim about $y$, we define the operator $\mathcal{C}: \mathcal{D}(\mathcal{A}) \rightarrow Y$ by

$$
\mathcal{C}\left[\begin{array}{l}
z \\
v
\end{array}\right]=\bar{C} z+D v(0)
$$

Then $\mathcal{C}$ is bounded from $\mathcal{D}(\mathcal{A})$ (with the graph norm) to $Y$. Now we show that $\mathcal{C}$ is an infinite-time admissible observation operator for $\mathcal{G}$. For $x_{0}, u$ and $y$ as in the proposition, we have (according to (3.8))

$$
y(t)=\bar{C} x(t)+D u(t)=\mathcal{C} \mathcal{G}_{t}\left[\begin{array}{c}
x_{0} \\
u
\end{array}\right],
$$

for all $t \geq 0$. Since $y$ is given by (3.6) and both $\Psi$ and $\mathbb{F}$ are (by assumptions (3) and (4)) bounded with range space $L^{2}([0, \infty), Y)$, we have $y \in L^{2}([0, \infty), Y)$ and

$$
\|y\|_{L^{2}} \leq k\left\|\left[\begin{array}{c}
x_{0} \\
u
\end{array}\right]\right\|_{X \times \mathcal{U}},
$$

with $k \geq 0$ independent of $x_{0}$ and $u$. This means that $\mathcal{C}$ is infinite-time admissible for the semigroup $\mathcal{G}$. Thus, $\mathcal{C}$ and $\mathcal{G}$ determine a bounded operator $\widetilde{\Psi}: X \times \mathcal{U} \rightarrow L^{2}([0, \infty), Y)$ similarly as in (2.7) and $y=\widetilde{\Psi} \xi_{0}$. The distributional derivative of $y$ is $\dot{y}(t)=\widetilde{\Psi} \mathcal{A} \xi_{0}$. Using the fact that $\mathcal{C}$ is infinite-time admissible, we conclude that $\dot{y} \in L^{2}([0, \infty), Y)$. Hence, $y \in \mathcal{H}^{1}(0, \infty ; Y)$, as claimed.

\section{The EQUations (1.7) AND (1.8) With $u \in \mathcal{H}^{2}$}

In this section we reformulate (1.7) as a first order equation on a product space and we study the solutions of (1.7) and (1.8) for $u \in H^{2}(0, \infty ; U)$ and compatible initial conditions. In particular, we show that a certain energy identity is satisfied, involving the input, the state and the output.

Notation. We use the notation $H, U, A_{0}, H_{\alpha}, B_{0}, C_{0}$, as introduced in Section 1 before (1.7). In particular, we recall that $A_{0}$ is positive and boundedly invertible on $H, C_{0} \in \mathcal{L}\left(H_{\frac{1}{2}}, U\right)$ and $B_{0}=C_{0}^{*}$. The duality is set up such that $H_{-\alpha}=H_{\alpha}^{*}$ and $U=U^{*}$. $A_{0}$ can be extended to all the spaces $H_{\alpha}$ and we have (1.6). The space $Z_{0}$ is defined as in Theorem 1.2. As in Section 1, we denote the open right half-plane by $\mathbb{C}_{0}$ and we define $X=H_{\frac{1}{2}} \times H$ and

$$
\begin{gathered}
A=\left[\begin{array}{cc}
0 & I \\
-A_{0}-\frac{1}{2} B_{0} C_{0}
\end{array}\right], \\
\mathcal{D}(A)=\left\{\left[\begin{array}{c}
z \\
w
\end{array}\right] \in H_{\frac{1}{2}} \times H_{\frac{1}{2}} \mid A_{0} z+\frac{1}{2} B_{0} C_{0} w \in H\right\} .
\end{gathered}
$$

Proposition 5.1. The operator $A$ is the generator of a strongly continuous contraction semigroup $\mathbb{T}$ on $X$.

This is well-known, see for example Triggiani [21] (for specific $A_{0}$ and $B_{0}$ ). We omit the details of the proof, but we give an outline. The first step is to show that for all $x \in \mathcal{D}(A)$ with second component $w$,

$$
\operatorname{Re}\langle A x, x\rangle=-\frac{1}{2}\left\|C_{0} w\right\|^{2}
$$

so that $A$ is dissipative. The second step is to show that $A$ is onto. Then it follows that $A$ is the generator of a contraction semigroup, see Pazy [13] (Sect. 1.4) or Bensoussan et al. [3] (Th. 2.7). 
We need to obtain some information about the space $X_{-1}$ (it seems that a simple characterization of this space is not possible). It is easy to check that

$$
A^{-1}=\left[\begin{array}{cc}
-\frac{1}{2} A_{0}^{-1} B_{0} C_{0} & -A_{0}^{-1} \\
I & 0
\end{array}\right] .
$$

Taking $\beta=0$ in the definition of $X_{-1}$ (Sect. 2), we conclude that $X_{-1}$ is the completion of $X$ with respect to the norm $\|\cdot\|_{-1}$ defined by

$$
\left\|\left[\begin{array}{c}
z \\
w
\end{array}\right]\right\|_{-1}^{2}=\left\|\frac{1}{2} A_{0}^{-\frac{1}{2}} B_{0} C_{0} z+A_{0}^{-\frac{1}{2}} w\right\|_{H}^{2}+\|z\|_{H}^{2} .
$$

Proposition 5.2. We have $H_{\frac{1}{2}} \times H_{-\frac{1}{2}} \subset X_{-1}$. Moreover, the embeddings

$$
X \subset H_{\frac{1}{2}} \times H_{-\frac{1}{2}} \subset X_{-1}
$$

are continuous.

Proof. It is clear that the first embedding in (5.5) holds and it is continuous. For $\left[\begin{array}{c}z \\ w\end{array}\right] \in X=H_{\frac{1}{2}} \times H$, we have

$$
\begin{aligned}
\left\|\left[\begin{array}{c}
z \\
w
\end{array}\right]\right\|_{-1}^{2} & \leq\left(\frac{1}{2}\left\|A_{0}^{-\frac{1}{2}} B_{0} C_{0} z\right\|_{H}+\left\|A_{0}^{-\frac{1}{2}} w\right\|_{H}\right)^{2}+\|z\|_{H}^{2} \\
& \leq\left(k_{1}\|z\|_{H_{\frac{1}{2}}}+\|w\|_{H_{-\frac{1}{2}}}\right)^{2}+\|z\|_{H}^{2} \leq k_{2}\left\|\left[\begin{array}{c}
z \\
w
\end{array}\right]\right\|_{H_{\frac{1}{2}} \times H_{-\frac{1}{2}}}^{2},
\end{aligned}
$$

where $k_{1}, k_{2}>0$. This estimate shows that $X_{-1}$, which is the completion of $X$ with respect to $\|\cdot\|_{-1}$, contains $H_{\frac{1}{2}} \times H_{-\frac{1}{2}}$, which can be thought of as the completion of $X$ with respect to $\|\cdot\|_{H_{\frac{1}{2}} \times H_{-\frac{1}{2}}}$. Moreover, it follows from the estimate that the second embedding in (5.5) is also continuous.

We now derive an explicit expression for $(s I-A)^{-1}$ extended to $H_{\frac{1}{2}} \times H_{-\frac{1}{2}}$ (this extension exists according to the previous proposition). Our computation is valid for all $s \in \rho(A)$, and since $A$ is a dissipative generator, $\mathbb{C}_{0} \subset \rho(A)$. The space $H_{\frac{1}{2}} \times H_{\frac{1}{2}}$ will play an important role in the sequel, and we note that

$$
X_{1} \subset H_{\frac{1}{2}} \times H_{\frac{1}{2}} \subset X
$$

Proposition 5.3. For every $s \in \rho(A)$ (in particular, for every $s \in \mathbb{C}_{0}$ ), the following statements hold:

(1) the operator $(s I-A)^{-1}$ is an isomorphism (i.e., a bounded and invertible map) between the spaces indicated here:

$$
(s I-A)^{-1}: H_{\frac{1}{2}} \times H_{-\frac{1}{2}} \rightarrow H_{\frac{1}{2}} \times H_{\frac{1}{2}}
$$

(2) the operator $s^{2} I+A_{0}+\frac{s}{2} B_{0} C_{0} \in \mathcal{L}\left(H_{\frac{1}{2}}, H_{-\frac{1}{2}}\right)$ has a bounded inverse

$$
V(s)=\left(s^{2} I+A_{0}+\frac{s}{2} B_{0} C_{0}\right)^{-1} \in \mathcal{L}\left(H_{-\frac{1}{2}}, H_{\frac{1}{2}}\right) ;
$$


(3) on $H_{\frac{1}{2}} \times H_{-\frac{1}{2}}$ we have, for every $s \in \rho(A)$ with $s \neq 0$,

$$
(s I-A)^{-1}=\left[\begin{array}{cc}
\frac{1}{s}\left[I-V(s) A_{0}\right] & V(s) \\
-V(s) A_{0} & s V(s)
\end{array}\right]
$$

(4) $V(s) B_{0} \in \mathcal{L}\left(U, Z_{0}\right)$, where $Z_{0}$ is the space defined in Theorem 1.2 .

Proof. We extend $(s I-A)^{-1}$ to $H_{\frac{1}{2}} \times H_{-\frac{1}{2}}$, which is possible according to Proposition 5.2, and clearly this extension maps into $X$, so that

$$
(s I-A)^{-1} \in \mathcal{L}\left(H_{\frac{1}{2}} \times H_{-\frac{1}{2}}, H_{\frac{1}{2}} \times H\right) .
$$

We partition this extended $(s I-A)^{-1}$ into a $2 \times 2$ matrix of operators corresponding to the two components of its domain space and its range space:

$$
(s I-A)^{-1}=\left[\begin{array}{ll}
Q(s) & V(s) \\
R(s) & W(s)
\end{array}\right]
$$

so that $Q(s) \in \mathcal{L}\left(H_{\frac{1}{2}}\right), V(s) \in \mathcal{L}\left(H_{-\frac{1}{2}}, H_{\frac{1}{2}}\right), R(s) \in \mathcal{L}\left(H_{\frac{1}{2}}, H\right), W(s) \in \mathcal{L}\left(H_{-\frac{1}{2}}, H\right)$. We rewrite the identity $(s I-A)(s I-A)^{-1}=I$ on $X=H_{\frac{1}{2}} \times H$ :

$$
\left[\begin{array}{cc}
s I & -I \\
A_{0} & s I+\frac{1}{2} B_{0} C_{0}
\end{array}\right] \cdot\left[\begin{array}{ll}
Q(s) & V(s) \\
R(s) & W(s)
\end{array}\right]=\left[\begin{array}{ll}
I & 0 \\
0 & I
\end{array}\right] .
$$

Looking at the $(1,2)$ entry, we see that $W(s)=s V(s)$ holds on $H$. By continuous extension, $W(s)=s V(s)$ remains valid on $H_{-\frac{1}{2}}$. Looking at the $(2,2)$ entry of $(5.9)$, we get that on $H, A_{0} V(s)+\left(s I+\frac{1}{2} B_{0} C_{0}\right) W(s)=I$. Combining this with our previous conclusion and extending by continuity to $H_{-\frac{1}{2}}$, we get

$$
\left(s^{2} I+A_{0}+\frac{s}{2} B_{0} C_{0}\right) V(s)=I \quad\left(\text { on } H_{-\frac{1}{2}}\right) .
$$

Looking at the $(1,1)$ entry of $(5.9)$, we see that on $H_{\frac{1}{2}}, s Q(s)-R(s)=I$, i.e.,

$$
Q(s)=\frac{1}{s}[I+R(s)] \quad\left(\text { on } H_{\frac{1}{2}}\right) .
$$

We have seen earlier that $Q(s) \in \mathcal{L}\left(H_{\frac{1}{2}}\right)$, which now implies that $R(s) \in \mathcal{L}\left(H_{\frac{1}{2}}\right)$. Together with $V(s) \in$ $\mathcal{L}\left(H_{-\frac{1}{2}}, H_{\frac{1}{2}}\right)$ and the formula $W(s)=s V(s)$, we now see that (5.6) holds. On the other hand, it is easy to see that

$$
s I-A: H_{\frac{1}{2}} \times H_{\frac{1}{2}} \rightarrow H_{\frac{1}{2}} \times H_{-\frac{1}{2}}
$$

and $s I-A$ is continuous between these spaces. Thus, $(s I-A)^{-1}$ is an isomorphism between the spaces shown in (5.6), as claimed in point (1) of the proposition.

Now let us rewrite the identity $(s I-A)^{-1}(s I-A)=I$ on $H_{\frac{1}{2}} \times H_{\frac{1}{2}}$ :

$$
\left[\begin{array}{cc}
\frac{1}{s}[I+R(s)] & V(s) \\
R(s) & s V(s)
\end{array}\right] \cdot\left[\begin{array}{cc}
s I & -I \\
A_{0} & s I+\frac{1}{2} B_{0} C_{0}
\end{array}\right]=\left[\begin{array}{ll}
I & 0 \\
0 & I
\end{array}\right] .
$$


Looking at the $(2,1)$ entry we get that on $H_{\frac{1}{2}}, R(s)=-V(s) A_{0}$. Substituting this into the last expression for $(s I-A)^{-1}$, we get that (5.8) holds on $H_{\frac{1}{2}} \times H_{-\frac{1}{2}}$, as claimed in point (3) (but we do not know yet that $V(s$ ) is given by (5.7)).

Looking at the $(2,2)$ entry of $(5.11)$, we get

$$
V(s)\left(s^{2} I+A_{0}+\frac{s}{2} B_{0} C_{0}\right)=I \quad\left(\text { on } H_{\frac{1}{2}}\right) .
$$

This, together with (5.10), implies that point (2) of the proposition also holds.

Finally, to prove point (4), we take $\mathrm{v} \in U$ and denote $z=V(s) B_{0} \mathrm{v}$. Then (5.10) applied to $B_{0} \mathrm{v}$ can be rewritten in the form

$$
A_{0} z=-s^{2} z+B_{0}\left(\mathrm{v}-\frac{s}{2} C_{0} z\right) .
$$

Applying $A_{0}^{-1}$ to both sides, we see that $z \in Z_{0}$. Moreover, using the definition of the norm on $Z_{0}$, we see that

$$
\|z\|_{Z_{0}}^{2} \leq\left\|s^{2} z\right\|_{H}^{2}+\left\|\mathrm{v}-\frac{s}{2} C_{0} z\right\|_{U}^{2} .
$$

This estimate easily implies that $A_{0}^{-1} B_{0}$ is bounded from $U$ to $Z_{0}$.

We define the operators

$$
B \in \mathcal{L}\left(U, H_{\frac{1}{2}} \times H_{-\frac{1}{2}}\right), \quad \bar{C} \in \mathcal{L}\left(Z_{0} \times H_{\frac{1}{2}}, U\right)
$$

as in Section 1, that is

$$
B=\left[\begin{array}{c}
0 \\
B_{0}
\end{array}\right], \quad \bar{C}=\left[\begin{array}{ll}
0 & -C_{0}
\end{array}\right]
$$

Note that, by Proposition 5.2, $B \in \mathcal{L}\left(U, X_{-1}\right)$. According to (5.8), for every $s \in \rho(A)$ with $s \neq 0$ (in particular, for every $s \in \mathbb{C}_{0}$ ),

$$
(s I-A)^{-1} B=V(s) B_{0}\left[\begin{array}{c}
I \\
s I
\end{array}\right], \quad \bar{C}(s I-A)^{-1}=C_{0} V(s)\left[\begin{array}{ll}
A_{0} & -s I
\end{array}\right] .
$$

In fact, equation (5.13) remains valid also for $s=0$, since from (5.4) we have

$$
A^{-1} B=\left[\begin{array}{c}
-A_{0}^{-1} B_{0} \\
0
\end{array}\right], \quad C A^{-1}=\left[\begin{array}{ll}
-C_{0} & 0
\end{array}\right] .
$$

Lemma 5.4. Let $u \in \mathcal{H}^{2}(0, \infty ; U)$ and $z_{0}, w_{0} \in H_{\frac{1}{2}}$ such that (1.9) holds. Then there exists a unique function

$$
z \in C\left(0, \infty ; Z_{0}\right) \cap C^{1}\left(0, \infty ; H_{\frac{1}{2}}\right) \cap C^{2}(0, \infty ; H)
$$

such that (1.11) holds and

$$
z(0)=z_{0}, \quad \dot{z}(0)=w_{0} .
$$

If we put $x_{0}=\left[\begin{array}{c}z_{0} \\ w_{0}\end{array}\right]$, then the function $x(t)=\left[\begin{array}{c}z(t) \\ \dot{z}(t)\end{array}\right]$ is given by (2.2) and it is the unique solution of $(2.1)$ in $X$, with $x(0)=x_{0}$.

Proof. We need the Hilbert space $Z$ introduced in (2.9) and (2.10). It is easy to see that in our specific framework,

$$
\mathcal{D}(A) \subset Z=Z_{0} \times H_{\frac{1}{2}} \subset H_{\frac{1}{2}} \times H_{\frac{1}{2}} .
$$


It is easy to check that, due to (1.9), $A x_{0}+B u(0) \in X$. We define the function $x$ by (2.2). According to Proposition 2.1, $x$ satisfies (2.1) in $X$ and $x \in C(0, \infty ; Z) \cap C^{1}(0, \infty ; X)$. From the structure of $A$ and $B$ we see that there exists a function $z$ such that $x(t)=\left[\begin{array}{c}z(t) \\ \dot{z}(t)\end{array}\right]$ and $z$ satisfies $(1.11,5.15)$ and (5.16).

Proposition 5.5. Under the assumptions and with the notation of Lemma 5.4, denoting $y(t)=-C_{0} \dot{z}(t)+u(t)$ (as in (1.12)), we have the identity

$$
\frac{\mathrm{d}}{\mathrm{d} t}\left\|\left[\begin{array}{c}
z(t) \\
\dot{z}(t)
\end{array}\right]\right\|_{H_{\frac{1}{2}} \times H}^{2}=\|u(t)\|_{U}^{2}-\|y(t)\|_{U}^{2}
$$

Proof. As we have seen in the proof of Lemma 5.4, the function $x \in C(0, \infty ; Z)$ defined by $x(t)=\left[\begin{array}{l}z(t) \\ \dot{z}(t)\end{array}\right]$ satisfies (2.1). Taking the scalar product of both sides in (2.1) with $x(t)$, we get

$$
\frac{1}{2} \frac{\mathrm{d}}{\mathrm{d} t}\|x(t)\|_{X}^{2}=\operatorname{Re}\langle A x(t)+B u(t), x(t)\rangle_{X} .
$$

If we replace $x(t)$ by $\left[\begin{array}{c}z(t) \\ \dot{z}(t)\end{array}\right]$ and use the structure of $A$ and $B$, we obtain

$$
\frac{1}{2} \frac{\mathrm{d}}{\mathrm{d} t}\left\|\left[\begin{array}{c}
z(t) \\
\dot{z}(t)
\end{array}\right]\right\|_{H_{\frac{1}{2}} \times H}^{2}=\operatorname{Re}\langle\dot{z}(t), z(t)\rangle_{H_{\frac{1}{2}}}+\operatorname{Re}\left\langle-A_{0} z(t)-\frac{1}{2} B_{0} C_{0} \dot{z}(t)+B_{0} u(t), \dot{z}(t)\right\rangle_{H} .
$$

We now replace the scalar product in $H$ (the last term above) by the duality pairing between $H_{-\frac{1}{2}}$ and $H_{\frac{1}{2}}$, which enables us to break it into three terms. Since $\operatorname{Re}\langle\dot{z}(t), z(t)\rangle_{H_{\frac{1}{2}}}=\operatorname{Re}\left\langle A_{0} z(t), \dot{z}(t)\right\rangle_{H}$ and $z$ satisfies (1.11), the last formula and the fact that $B_{0}^{*}=C_{0}$ imply that

$$
\frac{1}{2} \frac{\mathrm{d}}{\mathrm{d} t}\left\|\left[\begin{array}{c}
z(t) \\
\dot{z}(t)
\end{array}\right]\right\|_{H_{\frac{1}{2}} \times H}^{2}=-\frac{1}{2}\left\|C_{0} \dot{z}(t)\right\|_{U}^{2}+\operatorname{Re}\left\langle u(t), C_{0} \dot{z}(t)\right\rangle_{U} .
$$

Using now the simple formula

$$
\frac{1}{2}\|y(t)\|_{U}^{2}=\frac{1}{2}\left\|C_{0} \dot{z}(t)\right\|_{U}^{2}-\operatorname{Re}\left\langle u(t), C_{0} \dot{z}(t)\right\rangle_{U}+\frac{1}{2}\|u(t)\|_{U}^{2},
$$

we get the desired identity (5.17).

\section{Proof of the MAin Results}

In this section we prove Theorems 1.1-1.4 as well as other related results. We use the same assumptions and notation as in Section 5, including the operators $A$ defined in (5.1) and $B$ and $\bar{C}$ defined in (5.12). For every $\tau \geq 0$, we define the operator $\Phi_{\tau} \in \mathcal{L}\left(L^{2}([0, \infty), U), X_{-1}\right)$ as in (2.3), by $\Phi_{\tau} u=\int_{0}^{\tau} \mathbb{T}_{t-\sigma} B u(\sigma) \mathrm{d} \sigma$.

Proposition 6.1. $B$ is an infinite-time admissible control operator for $\mathbb{T}$. More precisely, $\Phi_{\tau} \in \mathcal{L}\left(L^{2}([0, \infty)\right.$, $U), X)$ holds for every $\tau \geq 0$ and

$$
\sup _{\tau \geq 0}\left\|\Phi_{\tau}\right\|_{\mathcal{L}\left(L^{2}([0, \infty), U), X\right)} \leq 1
$$


This implies that, for all $s \in \mathbb{C}_{0}$,

$$
\left\|s\left(s^{2} I+A_{0}+\frac{s}{2} B_{0} C_{0}\right)^{-1} B_{0}\right\|_{\mathcal{L}(U, H)} \leq \frac{1}{\sqrt{2 \operatorname{Re} s}} .
$$

Proof. Suppose first that $u \in \mathcal{H}_{L}^{2}(0, \infty ; U)=\left\{v \in \mathcal{H}^{2}(0, \infty ; U) \mid v(0)=0\right\}$ and define $x(t)=\Phi_{t} u$ for all $t \geq 0$. Then for any $\tau>0$, the restrictions of $u$ and $x$ to $[0, \tau]$ satisfy the assumptions of Proposition 2.1 , with $x_{0}=0$. This implies that $x$ is of the form $x=\left[\begin{array}{c}z \\ \dot{z}\end{array}\right]$ and $z$ satisfies $(1.11)$ and (5.16) with $z_{0}=w_{0}=0$. Integrating the identity in Proposition 5.5 on $[0, \tau]$, we get that for all $u \in \mathcal{H}_{L}^{2}(0, \infty ; U)$,

$$
\left\|\Phi_{\tau} u\right\|_{X}^{2} \leq \int_{0}^{\tau}\|u(t)\|_{U}^{2} \mathrm{~d} t \leq\|u\|_{L^{2}([0, \infty), U)}^{2}
$$

Since $\mathcal{H}_{L}^{2}(0, \infty ; U)$ is dense in $L^{2}([0, \infty), U)$, we obtain that $B$ is infinite-time admissible and $\left\|\Phi_{\tau}\right\| \leq 1$. This implies (using the operator $\tilde{\Phi}$ from Sect. 2) that

$$
\|\tilde{\Phi} u\|=\left\|\lim _{\tau \rightarrow \infty} \int_{0}^{\tau} \mathbb{T}_{t} B u(t) \mathrm{d} t\right\|_{X} \leq\|u\|_{L^{2}([0, \infty), U)}
$$

for all $u \in L^{2}([0, \infty), U)$. Taking $u(t)=\mathrm{e}^{-s t} u_{0}$, with $u_{0} \in U$ and $s \in \mathbb{C}_{0}$, we get

$$
\left\|(s I-A)^{-1} B u_{0}\right\|_{X} \leq \frac{1}{\sqrt{2 \operatorname{Re} s}}\left\|u_{0}\right\|_{U} .
$$

Since the second component of $(s I-A)^{-1} B$ is $s\left(s^{2} I+A_{0}+\frac{s}{2} B_{0} C_{0}\right)^{-1} B_{0}($ see $(5.13))$, we obtain the second estimate in the proposition.

We define $C$ as the restriction of $\bar{C}$ to $\mathcal{D}(A)$, so that $C \in \mathcal{L}\left(X_{1}, U\right)$. We define the output map $\Psi$ : $X_{1} \rightarrow L_{\mathrm{loc}}^{2}([0, \infty), U)$ by

$$
\left(\Psi x_{0}\right)(t)=C \mathbb{T}_{t} x_{0} \quad \forall x_{0} \in \mathcal{D}(A), \quad \forall t \geq 0
$$

The following result is dual to Proposition 6.1:

Proposition 6.2. The operator $C$ defined above is an infinite-time admissible observation operator for $\mathbb{T}$. More precisely,

$$
\int_{0}^{\infty}\left\|C \mathbb{T}_{t} x_{0}\right\|_{U}^{2} d t \leq\left\|x_{0}\right\|_{X}^{2} \quad \forall x_{0} \in \mathcal{D}(A),
$$

so that $\Psi$ can be extended to an operator $\Psi \in \mathcal{L}\left(X, L^{2}([0, \infty), U)\right)$ and we have $\|\Psi\|_{\mathcal{L}\left(X, L^{2}([0, \infty), U)\right)} \leq 1$. This implies that, for all $s \in \mathbb{C}_{0}$,

$$
\left\|C_{0}\left(s^{2} I+A_{0}+\frac{s}{2} B_{0} C_{0}\right)^{-1} A_{0}^{\frac{1}{2}}\right\|_{\mathcal{L}(H, U)} \leq \frac{1}{\sqrt{2 \operatorname{Re} s}} .
$$

Proof. Take $x_{0} \in \mathcal{D}(A)$ and put $x(t)=\mathbb{T}_{t} x_{0}$. Thus, $x$ is as in Lemma 2.1, with $u=0$. This implies that $x$ is of the form $x=\left[\begin{array}{c}z \\ \dot{z}\end{array}\right]$ and $z$ is the function described in Lemma 5.4, i.e., $z$ satisfies $(5.15,5.16)$ with $u=0$ and with $z_{0}$ and $w_{0}$ being the components of $x_{0}$. Integrating $(5.17)$ on $[0, \tau]$, we get

$$
\left\|x_{0}\right\|_{X}^{2}-\|x(\tau)\|_{X}^{2}=\|y\|_{L^{2}([0, \tau], U)}^{2}
$$


Note now that $y(t)=-C_{0} \dot{z}(t)=C x(t)$. Thus, the estimate

$$
\int_{0}^{\tau}\left\|C \mathbb{T}_{t} x_{0}\right\|_{U}^{2} \mathrm{~d} t \leq\left\|x_{0}\right\|_{X}^{2}
$$

holds for every $\tau \geq 0$. Obviously this implies the first estimate in the proposition. This first estimate implies, by the Cauchy-Schwarz inequality, that for every $s \in \mathbb{C}_{0}$ and for every $x_{0} \in \mathcal{D}(A)$

$$
\left\|\int_{0}^{\infty} \mathrm{e}^{-s t} C \mathbb{T}_{t} x_{0} \mathrm{~d} t\right\|_{U} \leq\left\|\mathrm{e}^{-s t}\right\|_{L^{2}}\left\|x_{0}\right\|_{X}=\frac{1}{\sqrt{2 \operatorname{Re} s}}\left\|x_{0}\right\|_{X} .
$$

Since the left-hand side above is $\left\|C(s I-A)^{-1} x_{0}\right\|$, using the first component of this operator (see (5.13)), we obtain that

$$
\left\|C_{0} V(s) A_{0}\right\|_{\mathcal{L}\left(H_{\frac{1}{2}}, U\right)} \leq \frac{1}{\sqrt{2 \operatorname{Re} s}} .
$$

Using that $A_{0}^{-\frac{1}{2}}$ is an isometric isomorphism from $H$ to $H_{\frac{1}{2}}$, we obtain the second estimate in the proposition.

Proof of Theorems 1.1 and 1.3. To prove Theorem 1.1, we have to show that the operators $A, B, \bar{C}$ together with $D=I$ define via (3.7) and (3.8) a conservative linear system $\Sigma$ which has the required properties $(1,2)$ and (3) listed in the theorem. Essentially, these properties mean that the system is described by (1.7) and (1.8), and these equations hold for every input function in $L^{2}$ and every initial state in $X$ (i.e., not merely for a dense space of smoother input functions and compatible initial states, like in Prop. 3.1). However, we remark that (3.7) and (3.8) will hold only for the smoother input functions and the compatible initial states, as described in the last part of Proposition 3.1. The proof of the two theorems cannot be separated: we have to prove gradually more and more facts from both theorems in order to make progress. Indeed, from the first step of the proof, we need the operators $A, B$ and $\bar{C}$, which do not appear in Theorem 1.1 at all, but are defined before Theorem 1.3. The proof of $C_{0} z \in \mathcal{H}^{1}(0, \infty ; U)$ (which is part of Th. 1.1) can be done only after Theorem 1.3 has been completely proved.

The first step is to verify that $A, B, \bar{C}$ and $D=I$ define via (3.7) and (3.8) a well-posed linear system $\Sigma$. This will follow from Proposition 3.1 if we check that all the assumptions of this proposition are true. We know from Proposition 5.1 that $A$ generates a contraction semigroup $\mathbb{T}$ on $X$. We know from Proposition 6.1 that $B$ is an admissible control operator for $\mathbb{T}$ (we actually know more from this proposition, but the other information is not needed now). Similarly, we know from Proposition 6.2 that $C$ is an admissible observation operator for $\mathbb{T}$. We define $\mathbb{F}$ by (3.9) and (3.10) (so that $\mathbb{F} u$ is defined for $u \in \mathcal{H}_{L}^{2}(0, \infty ; U)$ ). We consider the initial state to be $x_{0}=0$, i.e., we consider the state trajectory $x(t)=\Phi_{t} u$. Then all the assumptions of Lemma 5.4 are satisfied, so that according to this lemma, we have $x(t)=\left[\begin{array}{l}z(t) \\ \dot{z}(t)\end{array}\right]$, with the function $z$ satisfying (5.15) and (1.11). The function $y(t)=-C_{0} \dot{z}(t)+u(t)$ considered in Proposition 5.5 is the same as the function $y$ from (3.10). According to Proposition 5.5, we have for every $\tau \geq 0$

$$
\int_{0}^{\tau}\|y(t)\|^{2} \mathrm{~d} t=\int_{0}^{\tau}\|u(t)\|^{2} \mathrm{~d} t-\|x(\tau)\|^{2} .
$$

This shows that the estimate (3.11) is true for every $\tau \geq 0$, with $k=1$. According to Proposition 3.1, there exists a well-posed linear system $\Sigma$ with semigroup generator $A$, control operator $B$, observation operator $C$ and transfer function $\mathbf{G}$ given by $\mathbf{G}(s)=\bar{C}(s I-A)^{-1} B+I$. Still according to Proposition 3.1, if $u \in \mathcal{H}^{1}(0, \infty ; U)$, 
$x_{0} \in X, A x_{0}+B u(0) \in X$ and if we define $x$ and $y$ by

$$
x(t)=\mathbb{T}_{t} x_{0}+\Phi_{t} u, \quad y=\Psi x_{0}+\mathbb{F} u,
$$

then $x$ and $y$ satisfy (3.7) and (3.8) (with $D=I$ ), for every $t \geq 0$.

The second step is to show that for any $x_{0} \in X$ and any $u \in L^{2}([0, \infty), U)$, using the notation from (6.1) and denoting the first component of the state trajectory $x$ by $z$, we have $C_{0} z \in \mathcal{H}_{\text {loc }}^{1}(0, \infty ; U)$ and $(1.8)$ holds for almost every $t \geq 0$. (The space $\mathcal{H}_{\mathrm{loc}}^{1}(0, \infty ; U)$ has been introduced in Sect. 1.) We know from the end of the previous step that for certain $x_{0}$ and $u$, equations (3.7) and (3.8) hold for all $t \geq 0$. From the special structure of $A, B$ and $\bar{C}$ (see $(5.1,5.2)$ and (5.12)) we deduce that if $u \in \mathcal{H}^{1}(0, \infty ; U)$ and $A x_{0}+B u(0) \in X$, i.e., if

$$
x_{0}=\left[\begin{array}{c}
z_{0} \\
w_{0}
\end{array}\right] \quad \text { and } \quad A_{0} z_{0}+\frac{1}{2} B_{0} B_{0}^{*} w_{0}-B_{0} u(0) \in H,
$$

then the state trajectory $x$ can be decomposed as $x(t)=\left[\begin{array}{c}z(t) \\ \dot{z}(t)\end{array}\right]$ and $z, y$ satisfy (1.7) and (1.8) for all $t \geq 0$. By integrating (1.8), we see that if $u \in \mathcal{H}^{1}(0, \infty ; U)$ and the compatibility condition (6.2) holds, then for all $\tau \geq 0$

$$
C_{0} z(\tau)-C_{0} z(0)=\int_{0}^{\tau}[u(t)-y(t)] \mathrm{d} t .
$$

The left-hand side above depends continuously on $x(\tau)$ and on $x_{0}$ (considered as elements of $X=H_{\frac{1}{2}} \times H$ ). Thus, due to the well-posedness of $\Sigma$, both sides of (6.3) depend continuously on the pair $\left(x_{0}, u\right)$, considered as an element of $X \times L^{2}([0, \infty), U)$. Since the set of pairs $\left(x_{0}, u\right)$ considered in $(6.3)$ is dense in $X \times L^{2}([0, \infty), U)$, by continuous extension we obtain that (6.3) holds for every $x_{0} \in X$ and for every $u \in L^{2}([0, \infty), U)$. This implies that for every such $x_{0}$ and $u$,

$$
C_{0} z \in \mathcal{H}_{\mathrm{loc}}^{1}(0, \infty ; U)
$$

and (1.8) holds for almosty every $t \geq 0$, as claimed in point (3) of Theorem 1.1. (We shall see later that in fact, $C_{0} z \in \mathcal{H}^{1}(0, \infty ; U)$.)

The third step is to show that for every $x_{0} \in X$ and every $u \in L^{2}([0, \infty), U)$, denoting the two components of the state trajectory $x$ by $z$ and $w$, and denoting the output function by $y$, these functions have the properties stated in points (1) and (2) of Theorem 1.1 and moreover (1.7) holds for almost every $t \geq 0$. We know from the second step that if $u \in \mathcal{H}^{1}(0, \infty ; U)$ and $(6.2)$ holds, then $w=\dot{z}$ and $(1.7,1.8)$ hold for all $t \geq 0$. By integration we get the following equation in $H_{\frac{1}{2}}$ :

$$
z(\tau)-z(0)=\int_{0}^{\tau} w(t) \mathrm{d} t \quad \forall \tau \geq 0
$$

Due to the well-posedness of $\Sigma$, both sides of this equation depend continuously on the pair $\left(x_{0}, u\right)$, considered as an element of $X \times L^{2}([0, \infty), U)$. Using continuous extension, as we did for (6.3), we conclude that the above equation holds for every $x_{0} \in X$ and for every $u \in L^{2}([0, \infty), U)$. From the fact that $B$ is infinite-time admissible (Prop. 6.1) we know that $w \in B C(0, \infty ; H)$. Hence, $z \in C^{1}(0, \infty ; H)$ and $w=\dot{z}$ (we have proved point (2)). Moreover, using again the infinite-time admissibility of $B$, we see that $z \in B C\left(0, \infty ; H_{\frac{1}{2}}\right)$. Putting these facts together, we see that $z \in B C^{1}(0, \infty ; H)$. Now we substitute $C_{0} \dot{z}=u-y$ from (1.8) into (1.7) and we integrate, getting that for every $\tau \geq 0$,

$$
\dot{z}(\tau)-\dot{z}(0)=-A_{0} \int_{0}^{\tau} z(t) \mathrm{d} t+\frac{1}{2} B_{0} \int_{0}^{\tau}[u(t)+y(t)] \mathrm{d} t,
$$


which is an equation in $H_{-\frac{1}{2}}$. Again, both sides depend continuously on the pair $\left(x_{0}, u\right)$, considered as an element of $X \times L^{2}([0, \infty), U)$. Using continuous extension for the third time, we conclude that (6.4) holds for every $x_{0} \in X$ and for every $u \in L^{2}([0, \infty), U)$. Inspecting the terms on the right-hand side, we conclude that $\dot{z} \in \mathcal{H}_{\text {loc }}^{1}\left(0, \infty ; H_{-\frac{1}{2}}\right)$. This implies that $z \in \mathcal{H}_{\text {loc }}^{2}\left(0, \infty ; H_{-\frac{1}{2}}\right)$, as claimed in point (1). Now (6.4) together with (1.8) imply that (1.7) holds for almost every $t \geq 0$.

The fourth step is to show that $\Sigma$ is conservative and $\mathbf{G}$ is given by (1.14). We start by checking the formula for $\mathbf{G}$ : this is an easy consequence of the general formula (3.12) and of the formulas $(5.7,5.12)$ and (5.13). We know from Proposition 5.5 that if $u \in \mathcal{H}^{2}(0, \infty ; U)$ and (6.2) holds, then we have (5.17), which is the same as (4.2). By the converse part of Proposition 4.3, $\Sigma_{\tau}$ is isometric for every $\tau \geq 0$. Now consider the system $\Sigma^{d}$ obtained from $\Sigma$ by the state space isomorphism $J$ defined by the matrix

$$
J=\left[\begin{array}{cc}
I & 0 \\
0 & -I
\end{array}\right]
$$

i.e., by changing the sign of the second component of the state and leaving everything else unchanged. Thus, $\mathbb{T}_{t}^{d}=J \mathbb{T}_{t} J, \Phi_{t}^{d}=J \Phi_{t}, \Psi^{d}=\Psi J$ and $\mathbb{F}^{d}=\mathbb{F}$. Clearly, the transfer function of $\Sigma^{d}$ is $\mathbf{G}$, the same as for $\Sigma$. The generator of $\mathbb{T}^{d}$ is easily seen to be

$$
A^{d}=\left[\begin{array}{cc}
0 & -I \\
A_{0}-\frac{1}{2} B_{0} C_{0}
\end{array}\right]
$$

with

$$
\mathcal{D}\left(A^{d}\right)=\left\{\left[\begin{array}{c}
z \\
w
\end{array}\right] \in H_{\frac{1}{2}} \times H_{\frac{1}{2}} \mid-A_{0} z+\frac{1}{2} B_{0} C_{0} w \in H\right\},
$$

its control operator is $B^{d}=-B$ and its observation operator is $C^{d}=-C$. We claim that $\Sigma^{d}$ is in fact the dual system of $\Sigma$, as defined in Definition 4.1. It is easy to verify that for $x \in \mathcal{D}(A)$ and $x^{\prime} \in \mathcal{D}\left(A^{d}\right)$, we have $\left\langle A x, x^{\prime}\right\rangle=\left\langle x, A^{d} x^{\prime}\right\rangle$. Since $A$ and $A^{d}$ are generators, this shows that in fact, $A^{d}=A^{*}$. Similarly, it is easy to see that $B^{d}=C^{*}$ and $C^{d}=B^{*}$. Moreover, we have $\mathbf{G}(s)=\mathbf{G}(\bar{s})^{*}$, so that according to Proposition $4.2, \Sigma^{d}$ is the dual of $\Sigma$. From its definition it is clear that $\Sigma_{\tau}^{d}$ is isometric for every $\tau \geq 0$. Thus, by Corollary $4.4, \Sigma$ is conservative. In particular, it follows that for any initial state $x_{0} \in X$ and any input function $u \in L^{2}([0, \infty), U)$, the output function $y$ is in $L^{2}([0, \infty), U)$, as claimed in point (3) of Theorem 1.1.

The fifth step is to show that $\mathbf{G}$ has the properties stated in Theorem 1.3 and $C_{0} z \in \mathcal{H}^{1}(0, \infty ; U)$ (as claimed in point (3) of Th. 1.1). The fact that $\|\mathbf{G}(s)\| \leq 1$ for all $s \in \mathbb{C}_{0}$ follows from statement (4) in Proposition 4.5. Since $0 \in \rho(A)$, equation (3.12) shows that $\mathbf{G}$ has an analytic continuation to a neighborhood of 0 and now (1.14) implies (by taking limits from the right) that $\mathbf{G}(0)=I$. From (3.4) we see (by taking limits) that $\mathbf{G}^{\prime}(0)=$ $-\left(C A^{-1}\right)\left(A^{-1} B\right)$. Using (5.14) we obtain $\mathbf{G}^{\prime}(0)=-C_{0} A_{0}^{-1} B_{0}$. Similarly, the formula $\mathbf{G}^{\prime \prime}(0)=T^{2}$ is obtained from $\mathbf{G}^{\prime \prime}(0)=-2 C A^{-3} B$, using (5.4) and (5.14). Finally, to prove our claim concerning $C_{0} z$, consider $u, x_{0}, x$ and $y$ as in Theorem 1.1 and (just like in the theorem) denote the components of $x$ by $z$ and $w$. From the end of the previous step we know that $y \in L^{2}([0, \infty), U)$. Thus, according to (6.3), the derivative of $C_{0} z$ (in the sense of distributions on $(0, \infty))$ is in $L^{2}([0, \infty), U)$. To show that $C_{0} z \in \mathcal{H}^{1}(0, \infty ; U)$, it only remains to show that $C_{0} z \in L^{2}([0, \infty), U)$. To this end, we compute (using (5.14) and then (2.2))

$$
C_{0} z(t)=\left[\begin{array}{ll}
C_{0} & 0
\end{array}\right]\left[\begin{array}{c}
z(t) \\
w(t)
\end{array}\right]=-C A^{-1} x(t)=-C A^{-1} \mathbb{T}_{t} x_{0}-C A^{-1} \Phi_{t} u .
$$

The function $C \mathbb{T}_{t} A^{-1} x_{0}$ is in $L^{2}([0, \infty), U)$, because $C$ is infinite-time admissible. Thus, it only remains to show that the function $g(t)=C A^{-1} \Phi_{t} u$ is also in $L^{2}([0, \infty), U)$. The Laplace transform of $g$ is, according to (2.4) and (3.12),

$$
\hat{g}(s)=C A^{-1}(s I-A)^{-1} B \hat{u}(s)=\frac{1}{s}[\mathbf{G}(s)-\mathbf{G}(0)] \hat{u}(s) .
$$


Since $\mathbf{G}$ is bounded on $\mathbb{C}_{0}$ and it is analytic in a neighborhood of 0 , it follows that $\frac{1}{s}[\mathbf{G}(s)-\mathbf{G}(0)]$ is bounded on $\mathbb{C}_{0}$. By the Paley-Wiener theorem for the half-plane, $\hat{u}$ is in the Hardy space $H^{2}\left(\mathbb{C}_{0} ; U\right)$. Now we see from the formula of $\hat{g}(s)$ that this function is also in $H^{2}\left(\mathbb{C}_{0} ; U\right)$. Using again the Paley-Wiener theorem, we conclude that $g \in L^{2}([0, \infty), U)$.

Proof of Theorem 1.2. We already know from Theorem 1.1 that $\Sigma$ is a conservative linear system. This implies, according to Proposition 4.3, that for the differential energy balance equation (4.2) holds. Hence, according to Proposition 4.5, the four conditions in Proposition 4.6 are satisfied. Now, according to Proposition 4.6, for $u \in \mathcal{H}^{1}(0, \infty ; U)$ and $x_{0} \in X$ such that $A x_{0}+B u(0) \in X$, the state trajectory $x$ and the output function $y$ satisfy the smoothness and boundedness conditions (4.4) and they also satisfy the equations (3.7) and (3.8). The condition $A x_{0}+B u(0) \in X$ is, in our specific framework, using the formulas for $A$ and $B$ given after Theorem 1.2, equivalent to (1.9), where $z_{0}$ and $w_{0}$ are the components of $x_{0}$.

As already mentioned in Section 5 , it is easy to verify that the space $Z$ from (2.9) and (2.10) is given in our specific framework by $Z=Z_{0} \times H_{\frac{1}{2}}$. Using this fact, we can verify that the conditions (4.4) are, in our specific framework, equivalent to (1.10) together with the fact that $y \in \mathcal{H}^{1}(0, \infty ; U)$. Finally, the equations (3.7) and (3.8) are equivalent, in our specific framework, to (1.11) and (1.12), because in our framework $D=I$ (this follows from Th. 1.3 and (3.5)).

Proof of Theorem 1.4. If $z \in Z_{0}$ then $z=z_{1}+A_{0}^{-1} B_{0} v$, with $z_{1} \in H_{1}$ and $v \in U$. Applying $G_{0}$ we obtain $G_{0} z=v$, which shows that Ker $G_{0}=H_{1}$. Applying $G_{0} A_{0}^{-1}$ to both sides of the definition of $L_{0} z$, we obtain $G_{0} A_{0}^{-1} L_{0} z=0$, which shows that $A_{0}^{-1} L_{0} z \in H_{1}$, i.e., $L_{0} z \in H$. Since obviously $L_{0} \in \mathcal{L}\left(Z_{0}, H_{-\frac{1}{2}}\right)$, by the closed graph theorem we obtain $L_{0} \in \mathcal{L}\left(Z_{0}, H\right)$.

Let us show that (1.17) is equivalent to (1.9). If (1.9) holds, then applying $G_{0} A_{0}^{-1}$ to both sides we obtain straight (1.17). Conversely, if it is known that (1.17) holds, then we apply $B_{0}$ to both sides, obtaining $B_{0} G_{0} z_{0}$ $+\frac{1}{2} B_{0} C_{0} w_{0}-B_{0} u(0)=0$. Substituting $B_{0} G_{0}=A_{0}-L_{0}$ and using that $L_{0} z_{0} \in H$, we obtain (1.9).

Finally, the equivalence of (1.16) to (1.11) and (1.12) is proved by straightforward computations (start by applying $G_{0} A_{0}^{-1}$ to both sides of (1.11)).

Proof of Corollary 1.5. We know from Theorem 1.1 that $\Sigma$ is conservative. Since $u \in \mathcal{H}^{1}(0, \infty ; U)$ and $(1.9)$ holds, by Proposition 4.3 we have the differential energy balance equation (4.2). Using the formulas (1.16), this can be transformed into the equation that appears in the corollary.

Remark 6.3. With the assumptions and the notation of Theorem 1.4, if $v \in U, s \in \rho(A)$ and $x=(s I-A)^{-1} B v$, then $x=\left[\begin{array}{c}z \\ s z\end{array}\right]$, where $z \in Z_{0}$ satisfies

$$
\left(s^{2} I+L_{0}\right) z=0, \quad G_{0} z+\frac{s}{2} C_{0} z=v
$$

Indeed, this follows from $(s I-A) x=B v$, using the structure of $A$ and $B$, the definition of $L_{0}$ and the properties of $G_{0}$ postulated in the theorem.

\section{An eXAmple With the WAVE EQUation}

In this section we show that a certain infinite-dimensional system described by the wave equation on an $n$-dimensional domain, with mixed boundary control and mixed boundary observation, fits into the framework discussed in the previous sections. A somewhat simpler version of this system appears (as an example) also in the paper Weiss and Rebarber [26] (Sect. 7) and a related system is discussed in [14].

We assume that $\Omega \subset \mathbb{R}^{n}$ is a (possibly unbounded) domain with Lipschitz boundary $\Gamma$. Following the definition in Grisvard [6], this means that locally, after a suitable rotation and translation the coordinate system, the boundary is the graph of a Lipschitz function defined on a neighborhood of 0 in $\mathbb{R}^{n-1}$ (such a boundary admits corners and edges). $\Gamma_{0}$ and $\Gamma_{1}$ are nonempty open subsets of $\Gamma$ such that $\Gamma_{0} \cap \Gamma_{1}=\emptyset$ and 
$\overline{\Gamma_{0} \cup \Gamma_{1}}=\Gamma$. We denote by $x$ the space variable $(x \in \bar{\Omega})$. We assume that the Poincaré inequality holds for $\Omega$ and $\Gamma_{0}$. This means that there exists a $c>0$ such that for every $f \in \mathcal{H}^{1}(\Omega)$ with $\left.f\right|_{\Gamma_{0}}=0$,

$$
\int_{\Omega}\|(\nabla f)(x)\|^{2} \mathrm{~d} x \geq c \int_{\Omega}|f(x)|^{2} \mathrm{~d} x .
$$

This holds, in particular, if $\Omega$ is bounded. The Poincaré inequality holds also for certain unbounded domains, for example, if $\Omega$ is bounded in one direction and $\Gamma_{0}$ is large enough. A function $b \in L^{\infty}\left(\Gamma_{1}\right)$ is given, which intuitively expresses how strongly the input signal acts on different parts of the active boundary $\Gamma_{1}$. We assume that $b(x) \neq 0$ for almost every $x \in \Gamma_{1}$. The equations of the system are

$$
\begin{cases}\ddot{z}(x, t)=\Delta z(x, t) & \text { on } \Omega \times[0, \infty), \\ z(x, t)=0 & \text { on } \Gamma_{0} \times[0, \infty), \\ \frac{\partial}{\partial \nu} z(x, t)+|b(x)|^{2} \dot{z}(x, t)=\sqrt{2} \cdot b(x) u(x, t) & \text { on } \Gamma_{1} \times[0, \infty), \\ \frac{\partial}{\partial \nu} z(x, t)-|b(x)|^{2} \dot{z}(x, t)=\sqrt{2} \cdot b(x) y(x, t) & \text { on } \Gamma_{1} \times[0, \infty), \\ z(x, 0)=z_{0}(x), \quad \dot{z}(x, 0)=w_{0}(x) & \text { on } \Omega,\end{cases}
$$

where $u$ is the input function and $y$ is the output function. The functions $z_{0}$ and $w_{0}$ are the initial state of the system. The part $\Gamma_{0}$ of the boundary is just reflecting waves, while the active portion $\Gamma_{1}$ is where both the observation and the control take place. We may think of $u$ as the "incoming wave" (which brings energy into the system) and of $y$ as the "outgoing wave". We shall often write $z(t)$ to denote a function of $x$, meaning that $z(t)(x)=z(x, t)$, and similarly for other functions.

To put the equations (7.1) into the framework studied in this paper, we introduce the Hilbert spaces $H=$ $L^{2}(\Omega)$ and $U=L^{2}\left(\Gamma_{1}\right)$. The Dirichlet trace operator $\gamma$ is initially defined for any function $g \in C^{1}(\bar{\Omega})$ by

$$
\gamma g=\left.g\right|_{\Gamma} .
$$

If we regard $\gamma g$ as an element of $L^{2}(\Gamma)$, then the operator $\gamma$ has a continuous extension to $\mathcal{H}^{1}(\Omega)$. For the definition of the space $\mathcal{H}^{\frac{1}{2}}(\Gamma)$ (which is a dense subspace of $L^{2}(\Gamma)$ ) we refer again to Grisvard [6]. It is known that $\gamma$ maps $\mathcal{H}^{1}(\Omega)$ onto $\mathcal{H}^{\frac{1}{2}}(\Gamma)$ (see [6], Th. 1.5.1.3). We denote by $\mathcal{R}$ the usual restriction operator mapping $L^{2}(\Gamma)$ onto $L^{2}\left(\Gamma_{1}\right)$ and for all $g \in \mathcal{H}^{1}(\Omega)$ we put

$$
\gamma_{0} g=\mathcal{R} \gamma g .
$$

We call $\gamma_{0} g$ the Dirichlet trace of $g$ on $\Gamma_{1}$. If we regard $L^{2}\left(\Gamma_{1}\right)$ as a subspace of $L^{2}(\Gamma)$, then $I-\mathcal{R}$ is the restriction from $L^{2}(\Gamma)$ onto $L^{2}\left(\Gamma_{0}\right)$ and we define the Hilbert space

$$
\mathcal{H}_{\Gamma_{0}}^{1}(\Omega)=\left\{g \in \mathcal{H}^{1}(\Omega) \mid(I-\mathcal{R}) \gamma g=0\right\}, \quad\|g\|_{\mathcal{H}^{1}}=\|\nabla g\|_{H^{n}}
$$

We denote by $\widetilde{\mathcal{H}}^{\frac{1}{2}}\left(\Gamma_{1}\right)$ the space of all the traces $\gamma_{0} g$ with $g \in \mathcal{H}_{\Gamma_{0}}^{1}(\Omega)$. The norm of $v=\left.g\right|_{\Gamma_{1}}$ in the space $\widetilde{\mathcal{H}}^{\frac{1}{2}}\left(\Gamma_{1}\right)$ is the infimum of $\|g\|$ (the norm in $\mathcal{H}_{\Gamma_{0}}^{1}(\Omega)$ ) over all $g \in \mathcal{H}_{\Gamma_{0}}^{1}(\Omega)$ with the given trace $v$. Thus,

$$
\gamma_{0} \in \mathcal{L}\left(\mathcal{H}_{\Gamma_{0}}^{1}(\Omega), \widetilde{\mathcal{H}}^{\frac{1}{2}}\left(\Gamma_{1}\right)\right), \quad\left\|\gamma_{0}\right\| \leq 1
$$

The Neumann trace $\gamma_{1}$ is an operator originally defined on $C^{1}(\bar{\Omega})$ by

$$
\gamma_{1} f=\left.\frac{\partial}{\partial \nu} f\right|_{\Gamma_{1}}=\left.\langle\nabla f, \nu\rangle\right|_{\Gamma_{1}}
$$


where $\nu$ is the unit vector in the outward normal direction to $\Gamma_{1}$, which is defined almost everywhere on $\Gamma_{1}$. Thus, $\gamma_{1}$ is the outward normal derivative restricted to $\Gamma_{1}$. We will extend $\gamma_{1}$ using a version of Green's formula (see [7], p. 24) which says that for every $f \in \mathcal{H}^{2}(\Omega)$ and for every $g \in \mathcal{H}_{\Gamma_{0}}^{1}(\Omega)$,

$$
\left\langle\gamma_{1} f, \gamma_{0} g\right\rangle_{U}=\langle\Delta f, g\rangle_{H}+\langle\nabla f, \nabla g\rangle_{H^{n}} .
$$

(In [7], the formula is given for $g \in \mathcal{H}^{1}(\Omega)$, but we only need it for $g \in \mathcal{H}_{\Gamma_{0}}^{1}(\Omega)$.) Using this formula, we define $\gamma_{1} f$ for all those $f \in \mathcal{H}_{\Gamma_{0}}^{1}(\Omega)$ for which $\Delta f \in L^{2}(\Omega)$ ( $\Delta f$ is computed in the sense of distributions on $\Omega$ ). Indeed, the two terms on the right-hand side of Green's formula make sense for such $f$ and for any $g \in \mathcal{H}_{\Gamma_{0}}^{1}(\Omega)$. In this way, we obtain $\gamma_{1} f$ as an element of the dual space of $\widetilde{\mathcal{H}}^{\frac{1}{2}}\left(\Gamma_{1}\right)$, which we denote by $\widetilde{\mathcal{H}}^{-\frac{1}{2}}\left(\Gamma_{1}\right)$. It can be shown that $L^{2}\left(\Gamma_{1}\right) \subset \widetilde{\mathcal{H}}^{-\frac{1}{2}}\left(\Gamma_{1}\right)$, densely and with continuous embedding (this follows from the corresponding properties of $\widetilde{\mathcal{H}}^{\frac{1}{2}}\left(\Gamma_{1}\right)$ and $\left.L^{2}(\Gamma)\right)$.

Now we have all the ingredients needed to introduce the space

$$
Z_{0}=\left\{f \in \mathcal{H}_{\Gamma_{0}}^{1}(\Omega) \mid \Delta f \in L^{2}(\Omega), \gamma_{1} f \in b L^{2}\left(\Gamma_{1}\right)\right\}
$$

(the norm on $Z_{0}$ will be given later). Here, by some slight abuse of notation, $b$ denotes the operator of pointwise multiplication with the function $b$. We define the operator $A_{0}: \mathcal{D}\left(A_{0}\right) \subset L^{2}(\Omega) \rightarrow L^{2}(\Omega)=H$ by

$$
A_{0} z=-\Delta z, \quad \mathcal{D}\left(A_{0}\right)=\left\{z \in Z_{0} \mid \gamma_{1} z=0\right\} .
$$

Then $A_{0}$ is self-adjoint, positive and boundedly invertible (the bounded invertibility of $A_{0}$ follows from the Poincaré inequality). These and other details about $A_{0}$ can be found in Rodriguez-Bernal and Zuazua [14]. (The case when $\Omega$ is bounded, has $C^{2}$ boundary and $\overline{\Gamma_{0}} \cap \overline{\Gamma_{1}}=\emptyset$ has been discussed in Triggiani [21]. Under his assumptions, $Z_{0} \subset \mathcal{H}^{2}(\Omega)$, which makes things more manageable. The domain of $A_{0}$ was given incorrectly in Weiss and Rebarber [26], Sect. 7.)

The norms $\|z\|_{\alpha}$ and the spaces $H_{\alpha}$, with $\alpha \in \mathbb{R}$, are defined as in the Introduction. In particular, from Green's formula with $f=g \in \mathcal{D}\left(A_{0}\right)$ and continuous extension we obtain

$$
H_{\frac{1}{2}}=\mathcal{D}\left(A_{0}^{\frac{1}{2}}\right)=\mathcal{H}_{\Gamma_{0}}^{1}(\Omega)
$$

and

$$
\|z\|_{\frac{1}{2}}^{2}=\left\|A_{0}^{\frac{1}{2}} z\right\|_{H}^{2}=\int_{\Omega}\|\nabla z(x)\|^{2} \mathrm{~d} x .
$$

Proposition 7.1. The equations (7.1) determine a conservative linear system $\Sigma$ with input and output space $U$ and state space $H_{\frac{1}{2}} \times H$. If $z_{0} \in Z_{0}, w_{0} \in H_{\frac{1}{2}}, u \in \mathcal{H}^{1}(0, \infty ; U)$ and the compatibility condition

$$
\gamma_{1} z_{0}+|b|^{2} w_{0}=\sqrt{2} \cdot b u(0) \quad \text { on } \quad \Gamma_{1}
$$

holds, then (7.1) has a unique solution $z, y$ satisfying

$$
z \in B C\left(0, \infty ; Z_{0}\right) \cap B C^{1}\left(0, \infty ; H_{\frac{1}{2}}\right) \cap B C^{2}(0, \infty ; H), \quad y \in \mathcal{H}^{1}(0, \infty ; U) .
$$

Proof. There exists an operator $N \in \mathcal{L}\left(U, H_{\frac{1}{2}}\right)$, called the Neumann map, with the following property: $N v=g$ if and only if $g \in \mathcal{H}_{\Gamma_{0}}^{1}(\Omega), \Delta g=0$ and $\gamma_{1} g=v$. Indeed, by the Riesz representation theorem, for any $v \in U$ there exists a unique $g \in H_{\frac{1}{2}}=\mathcal{H}_{\Gamma_{0}}^{1}(\Omega)$ such that

$$
\langle g, \varphi\rangle_{H_{\frac{1}{2}}}=\left\langle v, \gamma_{0} \varphi\right\rangle_{U} \quad \forall \varphi \in \mathcal{H}_{\Gamma_{0}}^{1}(\Omega)=H_{\frac{1}{2}} .
$$


The left-hand side above can be written as $\langle\nabla g, \nabla \varphi\rangle_{H^{n}}$. By taking $\varphi \in C_{0}^{\infty}(\Omega)$, we see from the last formula together with Green's formula that $\Delta g=0$ (in the sense of distributions on $\Omega$ ). Hence, $\gamma_{1} g$ is well defined as an element of $\widetilde{\mathcal{H}}^{-\frac{1}{2}}\left(\Gamma_{1}\right)$ and, from $\langle\nabla g, \nabla \varphi\rangle=\left\langle v, \gamma_{0} \varphi\right\rangle$ we see that $\gamma_{1} g=v$.

We rewrite (7.2) in the form $\left\langle A_{0} N v, \varphi\right\rangle_{H}=\left\langle v, \gamma_{0} \varphi\right\rangle_{U}$, which shows that $N^{*} A_{0}=\gamma_{0}$. We define the operator $C_{0} \in \mathcal{L}\left(H_{\frac{1}{2}}, U\right)$ by

$$
C_{0}=\sqrt{2} \cdot \bar{b} N^{*} A_{0}=\sqrt{2} \cdot \bar{b} \gamma_{0} .
$$

Here, $\bar{b}$ is the operator of pointwise multiplication with the complex conjugate of the function $b$ introduced earlier. Thus, the adjoint of $C_{0}$ is

$$
B_{0}=C_{0}^{*}=\sqrt{2} \cdot A_{0} N b
$$

and $B_{0} \in \mathcal{L}\left(U, H_{-\frac{1}{2}}\right)$. The state space $X$ is defined, as in the Introduction, by $X=H_{\frac{1}{2}} \times H$, so that $X=$ $\mathcal{H}_{\Gamma_{0}}^{1}(\Omega) \times L^{2}(\Omega)$.

It is clear that the spaces $U, H, H_{\alpha}, X$ and the operators $A_{0}, C_{0}, B_{0}$ fit into the simple general framework of Section 1. Thus, by Theorems 1.1 and 1.3, they determine via the equations (1.7) and (1.8) a conservative linear system $\Sigma$ which has a list of special properties, described in the theorems. The state of this system is

$$
\xi(t)=\left[\begin{array}{c}
z(t) \\
\dot{z}(t)
\end{array}\right]
$$

(Note that we cannot use the letter $x$ for the state, as in earlier sections, since $x$ is now the space variable in $\Omega$.)

To show that $\Sigma$ is described by (7.1), first we notice that the space $Z_{0}$ introduced earlier in the example is the same as $Z_{0}=H_{1}+A_{0}^{-1} B_{0} U$ defined in Theorem 1.2. Thus, $Z_{0}$ is a Hilbert space with the norm defined in that theorem. We define the operator $G_{0} \in \mathcal{L}\left(Z_{0}, U\right)$ by

$$
G_{0}=\frac{1}{\sqrt{2}} \cdot b^{-1} \gamma_{1}
$$

Note that $b^{-1} \gamma_{1}$ cannot be defined on the larger space of those $f \in \mathcal{H}_{\Gamma_{0}}^{1}(\Omega)$ for which $\Delta f \in L^{2}(\Omega)$, but on $Z_{0}$, its definition makes sense because $\gamma_{1} f \in b L^{2}\left(\Gamma_{1}\right)$. Clearly we have $G_{0} H_{1}=\{0\}$ and

$$
G_{0} A_{0}^{-1} B_{0}=\frac{1}{\sqrt{2}} \cdot b^{-1} \gamma_{1} A_{0}^{-1} \sqrt{2} A_{0} N b=b^{-1} \gamma_{1} N b=I
$$

Hence, all the assumptions in Theorem 1.4 are satisfied. It follows from (7.3) that

$$
L_{0} z=A_{0} z-B_{0} G_{0} z=A_{0}\left(I-N \gamma_{1}\right) z=-\Delta z
$$

for all $z \in Z_{0}$, because $\left(I-N \gamma_{1}\right) z \in \mathcal{D}\left(A_{0}\right)$. If we write the system of equations (1.16) in our specific framework, we obtain the system of equations (7.1). Hence, by Theorem 1.4, the compatibility condition in our proposition is equivalent to (1.9) and the equations (7.1) are equivalent to (1.11) and (1.12). Now by Theorem 1.2 , the equations have a solution $z, y$ with the claimed smoothness properties.

Remark 7.2. We have shown that the equations (7.1) are equivalent to (1.11) and (1.12). These can be rewritten in the form (1.7) and (1.8), which make sense also for less smooth functions. Actually, by Theorem 1.1, the equations (1.7) and (1.8) (in the general case, and hence also in the particular context of our wave equation) admit a solution $z, y$ for any $z_{0} \in H_{\frac{1}{2}}, w_{0} \in H, u \in L^{2}([0, \infty), U)$, and the functions $z$ and $y$ will have the smoothness properties stated in Theorem 1.1. We may call those functions $z, y$ the generalized solutions of (7.1). 
The operator $A: \mathcal{D}(A) \rightarrow X$, the semigroup generator of the system $\Sigma$, is defined as in the text preceding Theorem 1.3 , by

$$
\begin{gathered}
A=\left[\begin{array}{cc}
0 & I \\
-A_{0}-\frac{1}{2} B_{0} C_{0}
\end{array}\right]=\left[\begin{array}{cc}
0 & I \\
-A_{0}-A_{0} N|b|^{2} N^{*} A_{0}
\end{array}\right], \\
\mathcal{D}(A)=\left\{\left[\begin{array}{c}
z \\
w
\end{array}\right] \in H_{\frac{1}{2}} \times\left. H_{\frac{1}{2}}|z+N| b\right|^{2} N^{*} A_{0} w \in H_{1}\right\} .
\end{gathered}
$$

It is interesting to note that we have the equivalent characterization

$$
A\left[\begin{array}{c}
z \\
w
\end{array}\right]=\left[\begin{array}{c}
w \\
\Delta z
\end{array}\right], \mathcal{D}(A)=\left\{\left[\begin{array}{c}
z \\
w
\end{array}\right] \underset{\mathcal{H}_{\Gamma_{0}}^{1}(\Omega)}{\stackrel{\mathcal{H}_{\Gamma_{0}}^{1}(\Omega)}{\times}} \mid \begin{array}{c}
\Delta z \in L^{2}(\Omega) \\
\gamma_{1} z+|b|^{2} \gamma_{0} w=0
\end{array}\right\}
$$

According to Proposition 5.1, $A$ generates a semigroup of contractions on $X$. The control operator $B$ and the observation operator $C$ of $\Sigma$ are given by

$$
B=\left[\begin{array}{c}
0 \\
B_{0}
\end{array}\right]=\left[\begin{array}{c}
0 \\
\sqrt{2} A_{0} N b
\end{array}\right], \quad C=\left[\begin{array}{ll}
0 & -C_{0}
\end{array}\right]=\left[\begin{array}{ll}
0 & -\sqrt{2} \bar{b} \gamma_{0}
\end{array}\right] .
$$

We think that the system $\Sigma$ is regular and its feedthrough operator is $D=0$. However, we do not have a rigorous proof of this statement.

The well-posedness of the equations (7.1), formulated in a different terminology (and for a different, but closely related system, without outputs and with $b=1$ ) is due to Rodriguez-Bernal and Zuazua [14].

Finally, consider the particular situation when $\Omega$ is one-dimensional and bounded: $\Omega=(0,1), \Gamma_{0}=\{0\}$, $\Gamma_{1}=\{1\}$, so that $H=L^{2}[0,1]$ and $U=\mathbb{C}$. Now the function $b$ becomes a nonzero number, and without loss of generality we may take $b=1$. We have $Z_{0}=\left\{f \in \mathcal{H}^{2}(0,1) \mid f(0)=0\right\}, \gamma_{0} g=g(1), \gamma_{1} f=f^{\prime}(1)$. It is easy to see (by solving the equations explicitly) that the input signal enters the domain at $x=1$, propagates along the domain (with unit speed) until it gets reflected at $x=0$ and then it propagates back to exit (as the output signal) at $x=1$. If the initial state is zero, then for $t \geq 2$ we have $y(t)=u(t-2)$, so that the transfer function is $\mathbf{G}(s)=\mathrm{e}^{-2 s}$. Note that $\mathbf{G}$ is regular with feedthrough operator zero.

This research was supported by EPSRC (from the UK) under grant number GR/R05048/01 and by CNRS (from France) under grant number 943706 DRCI. We have had useful discussions on this research with Olof Staffans, Arjan van der Schaft and Peng-Fei Yao.

\section{REFERENCES}

[1] D.Z. Arov and M.A. Nudelman, Passive linear stationary dynamical scattering systems with continous time. Integral Equations Operator Theory 24 (1996) 1-43.

[2] J.A. Ball, Conservative dynamical systems and nonlinear Livsic-Brodskii nodes. Oper. Theory Adv. Appl. 73 (1994) 67-95.

[3] A. Bensoussan, G. Da Prato, M.C. Delfour and S.K. Mitter, Representation and Control of Infinite Dimensional Systems, Vol. 1. Birkhäuser, Boston (1992).

[4] R.F. Curtain and G. Weiss, Well-posedness of triples of operators (in the sense of linear systems theory), Control and Estimation of Distributed Parameter Systems, edited by F. Kappel, K. Kunisch and W. Schappacher. Birkhäuser, Basel (1989) 41-59.

[5] P. Grabowski, On the spectral Lyapunov approach to parametric optimization of distributed parameter systems. IMA J. Math. Control Inform. 7 (1990) 317-338.

[6] P. Grisvard, Elliptic Problems in Nonsmooth Domains. Pitman, Boston (1985).

[7] P. Grisvard, Singularities in Boundary Value Problems. Masson, Paris (1992).

[8] S. Hansen and G. Weiss, New results on the operator Carleson measure criterion. IMA J. Math. Control Inform. 14 (1997) $3-32$.

[9] B. Jacob and J. Partington, The Weiss conjecture on admissibility of observation operators for contraction semigroups. Integral Equations Operator Theory (to appear). 
[10] P. Lax and R. Phillips, Scattering Theory. Academic Press, New York (1967).

[11] J.-L. Lions and E. Magenes, Non-Homogeneous Boundary Value Problems and Applications, Vol. I. Springer-Verlag, Berlin, Grundlehren Math. Wiss. 181 (1972).

[12] B.M.J. Maschke and A.J. van der Schaft, Portcontrolled Hamiltonian representation of distributed parameter systems, in Proc. of the IFAC Workshop on Lagrangian and Hamiltonian Methods for Nonlinear Control, edited by N.E. Leonard and R. Ortega. Princeton University (2000) 28-38.

[13] A. Pazy, Semigroups of Linear Operators and Applications to Partial Differential Equations. Springer-Verlag, New York (1983).

[14] A. Rodriguez-Bernal and E. Zuazua, Parabolic singular limit of a wave equation with localized boundary damping. Discrete Contin. Dynam. Systems 1 (1995) 303-346.

[15] D. Salamon, Infinite dimensional systems with unbounded control and observation: A functional analytic approach. Trans. Amer. Math. Soc. 300 (1987) 383-431.

[16] D. Salamon, Realization theory in Hilbert space. Math. Systems Theory 21 (1989) 147-164.

[17] O.J. Staffans, Quadratic optimal control of stable well-posed linear systems. Trans. Amer. Math. Soc. 349 (1997) $3679-3715$.

[18] O.J. Staffans, Coprime factorizations and well-posed linear systems. SIAM J. Control Optim. 36 (1998) 1268-1292.

[19] O.J. Staffans and G. Weiss, Transfer functions of regular linear systems. Part II: The system operator and the Lax-Phillips semigroup. Trans. Amer. Math. Soc. 354 (2002) 3229-3262.

[20] O.J. Staffans and G. Weiss, Transfer functions of regular linear systems. Part III: Inversions and duality (submitted).

[21] R. Triggiani, Wave equation on a bounded domain with boundary dissipation: An operator approach. J. Math. Anal. Appl. 137 (1989) 438-461.

[22] G. Weiss, Admissibility of unbounded control operators. SIAM J. Control Optim. 27 (1989) 527-545.

[23] G. Weiss, Admissible observation operators for linear semigroups. Israel J. Math. 65 (1989) 17-43.

[24] G. Weiss, Transfer functions of regular linear systems. Part I: Characterizations of regularity. Trans. Amer. Math. Soc. 342 (1994) 827-854.

[25] G. Weiss, Regular linear systems with feedback. Math. Control Signals Systems 7 (1994) 23-57.

[26] G. Weiss and R. Rebarber, Optimizability and estimatability for infinite-dimensional linear systems. SIAM J. Control Optim. 39 (2001) 1204-1232.

[27] G. Weiss, O.J. Staffans and M. Tucsnak, Well-posed linear systems - A survey with emphasis on conservative systems. Appl. Math. Comput. Sci. 11 (2001) 101-127. 